\title{
The acoustic Green's function for swirling flow with variable entropy in a lined duct
}

\author{
J.R. Mathews ${ }^{\mathrm{a}, *}$, N. Peake ${ }^{\mathrm{a}}$ \\ ${ }^{a}$ Department of Applied Mathematics and Theoretical Physics, University of Cambridge, Cambridge.
}

\begin{abstract}
This paper extends previous work by the authors (Journal of Sound and Vibration, 395:294-316,2017) on the acoustic field inside an annular duct with acoustic lining carrying mean axial and swirling flow so as to allow for non-uniform mean entropy, as would be found for instance in the turbine stage of an aeroengine. The main aim of this paper is to understand the effect of a non-uniform entropy on both the eigenmodes of the flow and the Green's function, which will allow noise prediction once we have identified acoustic sources. We first derive a new acoustic analogy in isentropic swirling flow, which allows us to derive the equation the tailored Green's function satisfies. The eigenmodes are split into two distinct families, acoustic and hydrodynamic modes, and are computed using different analytical methods; in the limit of high reduced frequency using the WKB method for the acoustic modes; and by considering a Frobenius expansion for the hydrodynamic modes. These are then compared with numerical results, with excellent agreement for all eigenmodes. The Green's function is also calculating analytically using the realistic limit of high reduced frequency, again with excellent agreement compared to numerical calculations. We see that for both the eigenmodes and Green's function the effect of non-uniform mean entropy is significant.
\end{abstract}

Keywords: Aeroacoustics, turbomachinery, swirl, entropy, Green's function, high-frequency asymptotics

\section{Introduction}

In light of increasing environmental concerns, operational targets and airport expansion it is vital to understand and model sound levels produced by aircraft engines accurately. One key noise source of the aircraft engine comes from the rotor and its interaction with the stator, for which understanding the propagation of acoustic waves through swirling, isentropic flow in an annular duct will be crucial and which we focus on in this paper. This analysis will also be useful in the turbine and compressor regions of the aeroengine, where the mean entropy is often non-uniform.

To calculate the propagation of noise in an aeroengine an acoustic analogy is often required. Lighthill 21] was the first to derive an acoustic analogy, famously rearranging the Navier-Stokes equations into a single equation, with the left-hand side the wave operator in a stationary fluid acting on the density perturbation. The right-hand side of the analogy consists of the remaining terms (including non-linear and viscous effects), and is thought of as the sound source. If the right-hand side is known, then the solution of Lighthill's analogy is given by a convolution of the source terms and the free space Green's function of the wave operator. Lighthill's analogy has been extended in numerous ways; Curle [8] and Ffowcs Williams and Hawkings [12] considered moving surfaces, while Goldstein [13] and Morfey and Wright [28] considered different dependent variables on the left-hand side. Often, the fluid is not stationary, and has a radially varying base flow, which requires a new acoustic analogy. Lilley 22 extended the acoustic analogy to flows with a unidirectional base shear flow, and the left-hand side of his operator is often approximated by the

\footnotetext{
* Corresponding author

Email addresses: jrm214@cam.ac.uk (J.R. Mathews), np100@cam.ac.uk (N. Peake)
} 
linear Pridmore-Brown equation [33] acting on the logarithm of the pressure perturbation. Finally, Posson and Peake 32 considered a swirling base flow in a duct and derived a sixth order linear operator acting on the pressure perturbation. For all of these acoustic analogies it will be necessary to find the Green's function of the left-hand side, and the complexity of this Green's function increases with the complexity of the base flow.

Once we have calculated the Green's function, we can predict the noise generated for simple source distributions, as performed by Posson and Peake 32 and Masson et al. 23] for swirling flow. Alternatively, the Green's function has applications in many other noise prediction methods such as beamforming and the two-microphone method. Beamforming is now one of the major processing tools to analyse data microphone array data in aeroengine noise tests, and the Green's function is used to infer information about the noise sources from the measurements. Although much recent progress has been made, such as by Sijtsma 35, the most state-of the art base flow currently being used is a piecewise constant unidirectional shear flow 36]. Another potential application for the Green's function in swirling flow is the two-microphone method, which uses the Green's function to determine how noise propagates between two microphone measurements. It is currently being worked on by Joseph et al. 18, Chen and Joseph [4, but at present is restricted to a uniform axial base flow.

To evaluate the Green's function, we need to firstly calculate the eigenmodes of the linearised Euler equations about a swirling, isentropic base flow. These eigenmodes can be split into two distinct families. The acoustic modes are pressure-driven, while the hydrodynamic (or nearly convected) modes contain most of the unsteady vorticity of the flow. There is also the critical layer, which is a singularity of the system of linearised Euler equations. We can get rid of this artefact by including higher order terms or viscosity, but most of the time the effect of the critical layer is neglected since it is assumed to be small [32. Once we have calculated the eigenmodes, we find the Green's function by evaluating the residues of the acoustic eigenmodes, and neglecting the contributions from the hydrodynamic modes and critical layers.

The hydrodynamic modes were first shown to exist by Kerrebrock [19]. It was shown that there are infinitely many of them when we consider swirling mean flow by Golubev and Atassi [14, who were among the first to study the asymptotic behaviour of these modes. A further numerical study of the modes was performed by Nijboer [30. The first full asymptotic treatment of the hydrodynamic modes was done by Heaton and Peake [17, who showed three possible asymptotic regimes of the hydrodynamic modes, depending on the flow parameters. It was shown that the modes could accumulate either exponentially or algebraically, with the latter splitting into two cases, on the real line and in the complex plane. Heaton and Peake's work addressed several issues with earlier work such as the use of a thin duct assumption in Golubev and Atassi 14 .

There have been numerous studies about the acoustic eigenmodes. In Cooper and Peake [6], Heaton and Peake [16] the acoustic eigenmodes and eigenfunctions for swirling flow in a hard-walled infinite duct were calculated asymptotically, using the WKB method. In both papers they show we can get turning points in the WKB method, corresponding physically to caustics, Cooper and Peake [6, Figure 10]. In Vilenski and Rienstra [39, 38, a lined infinite duct is considered, but for zero swirl. They seek the acoustic eigenmodes and eigenfunctions of the resulting Pridmore-Brown 33 differential equation, and compare their asymptotic method with some numerical results. In Posson and Peake 32 the eigenmodes and Green's functions were calculated numerically for swirling flow with constant entropy in a hard-walled infinite duct. In Posson and Peake 31] the results were extended to an infinite duct with acoustic lining. In Mathews and Peake [26] the eigenmodes and Green's functions were calculated analytically for swirling flow and compared to the numerical results, showing excellent agreement, even for the cases of semi-realistic swirling flow in a lined duct.

Relatively little work has been carried out on the effect of entropy on the eigenmodes and Green's function, although Tam and Auriault [37] considered a isentropic flow. They calculate eigenmodes and a Green's function in the case of simple swirling flow in an infinite hard-walled duct, and their choice of base flow density ensured the entropy of the base flow varied. In Cooper [5] the effect of entropy is considered on the propagation of the pressure field in a slowly varying duct, with most of the analysis only concerning the first cut-on eigenmode and associated eigenfunction. 


\subsection{Organisation}

This paper is laid out as follows. In Section 2 we derive a new acoustic analogy, based on the work from Posson and Peake 32. In Posson and Peake 32. the base flow used in the acoustic analogy is homentropic, while we allow the entropy to vary radially in the base flow. In Section 3 we revisit the asymptotic and numerical methods for finding the acoustic eigenmodes and Green's function in swirling, isentropic flow, with the methods based upon Mathews and Peake [26]. In Section 4 we consider the effect of entropy on the acoustic eigenmodes, and show that there are three main features due to the variable entropy. In Section 5 we consider the effect of entropy on the hydrodynamic modes, both numerically and asymptotically. We extend the work from Heaton and Peake [17] to an isentropic flow, and find there are still three accumulation regimes. Finally, in Section 6 we consider the effect of entropy on the Green's function, which is calculated by evaluating the residue at each of the acoustic modes.

\section{Acoustic analogy in isentropic swirling flow}

We will model the aeroengine as an infinite cylindrical duct, and in Figure 1 we see the cylindrical coordinate system, with $x$ the axial coordinate, $r$ the radial coordinate and $\theta$ the azimuthal coordinate. We let $\underline{u}, \underline{v}$, and $\underline{w}$ be the velocities in the $x, r$ and $\theta$ directions respectively. We split the inviscid, compressible, isentropic total flow (underlined) of air into a base flow (subscript 0) plus some small timeharmonic perturbations, so we have

$$
(\underline{u}, \underline{v}, \underline{w}, \underline{\rho}, \underline{p}, \underline{s})=\left(u_{0}, v_{0}, w_{0}, \rho_{0}, p_{0}, s_{0}\right)+(u, v, w, \rho, p, s),
$$

where $\underline{\boldsymbol{u}}=(\underline{u}, \underline{v}, \underline{w})$ is the total velocity of the air, $\underline{\rho}$ is the total density, $\underline{p}$ the total pressure and $\underline{s}$ the total entropy.

Let the inner and outer duct walls be given by $r^{\ddagger}=h^{\ddagger}$ and $r^{\ddagger}=d^{\ddagger}$ respectively, where the double dagger $\ddagger$ represents dimensioned coordinates. We non-dimensionalise all distances by $d^{\ddagger}$, so that the inner wall lies at $r=h:=h^{\ddagger} / d^{\ddagger}$ and the outer wall at $r=1$. We non-dimensionalise all velocities by the speed of sound at $r=d^{\ddagger}, c_{0}^{\ddagger}\left(d^{\ddagger}\right)$. Finally, we non-dimensionalise time by $d^{\ddagger} / c_{0}^{\ddagger}\left(d^{\ddagger}\right)$ and all frequencies by $c_{0}^{\ddagger}\left(d^{\ddagger}\right) / d^{\ddagger}$.

\subsection{Euler equations}

Let us next consider the motion of an inviscid, compressible, isentropic fluid, which is described by the Euler equations,

$$
\begin{array}{r}
\frac{\partial \underline{\rho}}{\partial t}+\nabla \cdot(\underline{\rho} \underline{\boldsymbol{u}})=0, \\
\underline{\rho}\left(\frac{\partial \underline{\boldsymbol{u}}}{\partial t}+\underline{\boldsymbol{u}} \cdot \nabla \underline{\boldsymbol{u}}\right)+\nabla \underline{p}=0, \\
\partial \underline{p}{ }^{\underline{t}}+\underline{\boldsymbol{u}} \cdot \nabla \underline{p}+\gamma \underline{p}(\nabla \cdot \underline{\boldsymbol{u}})=0 .
\end{array}
$$

Our choice of energy equation is derived by using the ideal gas law and the perfect gas law, where $\gamma$ is the ratio of the constant specific heat capacities $c_{p}$ and $c_{v}(\gamma=1.4$ for air $)$.

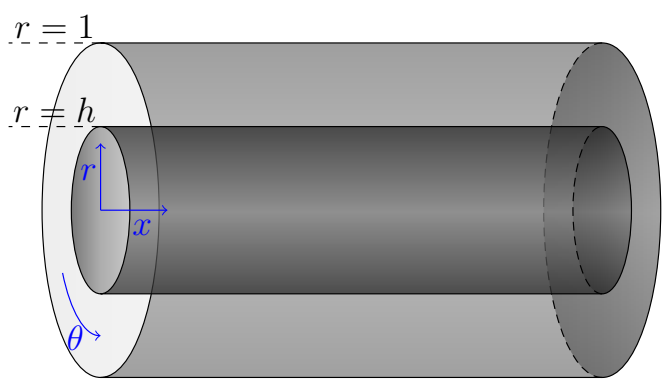

Figure 1: Geometry of the duct. 


\subsection{Swirling base flow}

The base flow is a solution to the Euler equations, with the base flow entropy $s_{0}$ satisfying the constitutive relation

$$
s_{0}=c_{v} \log \left(\frac{\gamma p_{0}}{\rho_{0}^{\gamma}}\right)
$$

while the speed of sound $c_{0}$ is defined from the Newton-Laplace equation, giving

$$
c_{0}^{2}=\frac{\gamma p_{0}}{\rho_{0}} .
$$

We define the base flow by specifying the base flow velocities and entropy, and then calculate the pressure, density and speed of sound from the above equations. We choose base flow velocities of the form

$$
\left(u_{0}, v_{0}, w_{0}\right)=\left(U_{x}(r), 0, U_{\theta}(r)\right),
$$

where $U_{x}(r)$ and $U_{\theta}(r)$ are freely chosen and setting the radial baseflow to zero, which is a reasonable assumption of the mean flow between the rotor and stator, as discussed in Mathews and Peake [26. We also specify that the base flow entropy only depends on the radial position; $s_{0}(r)$. We choose the pressure so that the Euler equations are satisfied, which essentially comes down to conservation of angular momentum, with

$$
p_{0}^{\prime}(r)=\frac{\rho_{0}(r) U_{\theta}^{2}(r)}{r} \text { or } p_{0}(r)=p_{0}(1)+\int_{1}^{r} \frac{\rho_{0}(s) U_{\theta}^{2}(s)}{s} \mathrm{~d} s .
$$

To determine $c_{0}(r)$ and $\rho_{0}(r)$ we first differentiate the two relations in Eq. (5) and Eq. (6), and use Eq. (8) to give (where primes denote differentiation with respect to $r$ throughout the paper)

$$
\frac{c_{0}^{2}(r)}{c_{0}^{2}(r)}=\frac{(\gamma-1) U_{\theta}^{2}(r)}{r c_{0}^{2}(r)}+\frac{s_{0}^{\prime}(r)}{c_{p}}
$$

We solve this differential equation by using an integrating factor (of $\exp \left[s_{0}(r) / c_{p}\right]$ ), which gives

$$
c_{0}^{2}(r)=c_{0}^{2}(1) \exp \left(\frac{s_{0}(r)-s_{0}(1)}{c_{p}}\right)+(\gamma-1) \exp \left(\frac{s_{0}(r)}{c_{p}}\right) \int_{1}^{r} \exp \left(-\frac{s_{0}(s)}{c_{p}}\right) \frac{U_{\theta}^{2}(s)}{s} \mathrm{~d} s .
$$

We can also get a first order differential equation for $\rho_{0}(r)$, which we solve by using an integrating factor of $\exp \left((\gamma-1) s_{0}(r) / c_{p}\right)$, and hence we find that

$$
\rho_{0}^{\gamma-1}(r)=\rho_{0}^{\gamma-1}(1) \exp \left(\frac{\gamma-1}{c_{p}}\left(s_{0}(1)-s_{0}(r)\right)\right)+(\gamma-1) \exp \left(\frac{1-\gamma}{c_{p}} s_{0}(r)\right) \int_{1}^{r} \exp \left(-\frac{s_{0}(s)}{c_{p}}\right) \frac{U_{\theta}^{2}(s)}{s} \mathrm{~d} s .
$$

We thus find the density is given by

$$
\rho_{0}(r)=\left[c_{0}^{2}(r) \exp \left(-\frac{s_{0}(r)}{c_{v}}\right)\right]^{1 /(\gamma-1)} .
$$

We consider several different choices of base flow entropy, a one parameter family of base flow entropies from Cooper [5], constant entropy and entropy such that the speed of sound is constant.

\subsubsection{Logarithmic entropy}

Cooper [5] suggests using a base flow entropy of the form

$$
s_{0}(r)=-\log \left(r^{\beta}\right),
$$




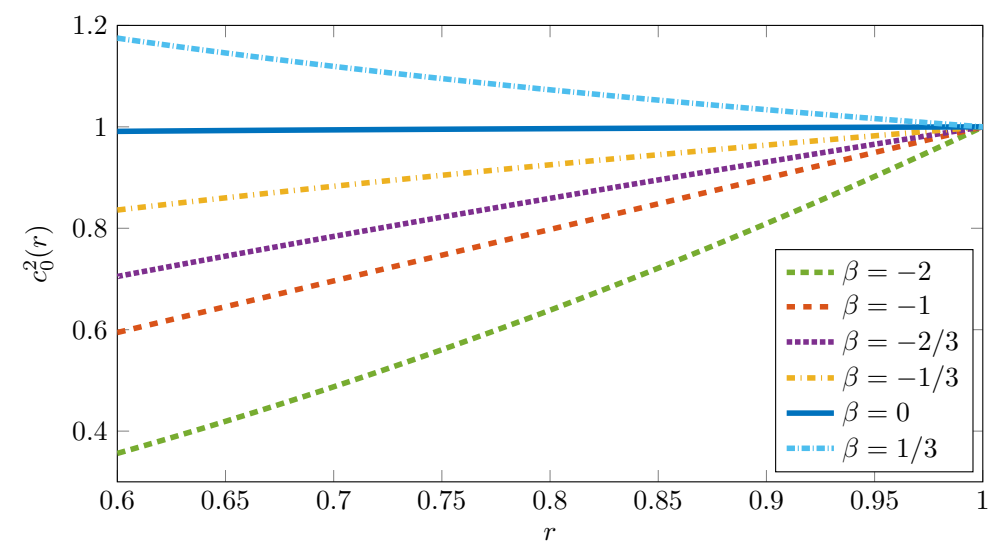

Figure 2: Plot of $c_{0}^{2}(r)$ against $r$ for different values of $\beta$ for the logarithm entropy $s_{0}(r)=-\log \left(r^{\beta}\right)$ and the swirling flow $U_{\theta}(r)=0.1 r+0.1 / r$.

where $\beta$ is a constant, which was chosen to simplify the governing equations she was considering. Using the previous section, we see that the speed of sound and pressure satisfy

$$
c_{0}^{2}(r)=c_{0}^{2}(1) r^{-\beta / c_{p}}+(\gamma-1) r^{-\beta / c_{p}} \int_{1}^{r} s^{\beta / c_{p}} \frac{U_{\theta}^{2}(s)}{s} \mathrm{~d} s \text { and } \rho_{0}^{\gamma-1}(r)=r^{\beta / c_{v}} c_{0}^{2}(r) .
$$

Note that the entropy is only defined up to an arbitrary constant, and varying the constant does not change the base flow speed of sound or density. In Figure 2 we plot how the speed of sound $c_{0}^{2}(r)$ varies with $r$ for different values of $\beta$ for a swirling flow of the form $U_{\theta}(r)=0.1 r+0.1 / r$. For all values of $\beta$ we still have $c_{0}^{2}(1)=1$ due to our non-dimensionalisation, but the entropy significantly changes how much the speed of sound varies in the duct. For negative $\beta$ we reduce the value of $c_{0}^{2}(h)$, from $c_{0}^{2}(h)=0.991$ (to 3 decimal places) when $\beta=0$, to $c_{0}^{2}(h)=0.836$ when $\beta=-1 / 3$ and $c_{0}^{2}(h)=0.594$ when $\beta=-1$. If we increase $\beta$ then we increase the value of $c_{0}^{2}(h)$, which can become greater than 1 , for example $c_{0}^{2}(h)=1.175$ when $\beta=1 / 3$. The local Mach number therefore varies with radius in a complicated way.

\subsubsection{Constant entropy}

For the homentropic case of Posson and Peake [32], $\beta=0$, and we calculate the speed of sound and density as

$$
c_{0}^{2}(r)=c_{0}^{2}(1)+(\gamma-1) \int_{1}^{r} \frac{U_{\theta}^{2}(s)}{s} \mathrm{~d} s \text { and } \rho_{0}^{\gamma-1}(r)=c_{0}^{2}(r) .
$$

\subsubsection{Constant speed of sound}

A final choice of entropy is such that the mean speed of sound is constant, which is equivalent to constant mean temperature for an ideal gas. From Eq. (9) it is clear that the required entropy profile is

$$
s_{0}(r)=-\frac{c_{p}(\gamma-1)}{c_{0}^{2}(1)} \int_{1}^{r} \frac{U_{\theta}^{2}(s)}{s} \mathrm{~d} s,
$$

up to a constant. Although the speed of sound is constant, the density still varies radially due to Eq. 12.

\subsection{Acoustic lining of the duct}

To mathematically model the acoustic lining we introduce the impedances $Z_{h}, Z_{1} \in \mathbb{C}$ of the liner at the duct walls. We non-dimensionalise the impedances by $Z_{j}=Z_{j}^{\ddagger} c_{0}^{\ddagger}\left(d^{\ddagger}\right) \rho_{0}^{\ddagger}\left(d^{\ddagger}\right)$, and assume that these impedances are uniform. The boundary conditions for the unsteady flow are then the standard Myers boundary conditions [29, allowing for slip flow at the duct walls

$$
\begin{aligned}
\mathrm{i} \omega v & =\left(-\mathrm{i} \omega+U_{x} \frac{\partial}{\partial x}+\frac{U_{\theta}}{r} \frac{\partial}{\partial \theta}\right)\left(\frac{p}{Z_{h}}\right) \text { on } r=h, \\
-\mathrm{i} \omega v & =\left(-\mathrm{i} \omega+U_{x} \frac{\partial}{\partial x}+\frac{U_{\theta}}{r} \frac{\partial}{\partial \theta}\right)\left(\frac{p}{Z_{1}}\right) \text { on } r=1 .
\end{aligned}
$$


Here and throughout we assume the time dependence of the flow is of the form $\exp (-\mathrm{i} \omega t)$. For rigid walls we have $Z_{h}=Z_{1}=\infty$ and hence the the boundary conditions become the no-penetration conditions $v(h)=v(1)=0$. We note that since the impedance $Z_{h}$ is non-dimensionalised by the outer wall, varying the mean flow entropy causes the effective impedance to vary at the inner wall, even though $Z_{h}$ is constant.

In Masson et al. 24 it was shown in arbitrary swirling flow the Myers boundary condition is not the correct boundary condition in the limit of an infinitely boundary layer. In non-swirling flow, Eversman and Beckemeyer [10] showed the Myers boundary condition was correct in the limit of an infinitely boundary layer. Using the results from Masson et al. 24] gives the boundary condition as

$$
\begin{aligned}
\mathrm{i} \omega v & =\left(-\mathrm{i} \omega+U_{x} \frac{\partial}{\partial x}+\frac{U_{\theta}}{r} \frac{\partial}{\partial \theta}\right)\left(\frac{p}{Z_{h}^{\star}}\right) \text { on } r=h, \\
-\mathrm{i} \omega v & =\left(-\mathrm{i} \omega+U_{x} \frac{\partial}{\partial x}+\frac{U_{\theta}}{r} \frac{\partial}{\partial \theta}\right)\left(\frac{p}{Z_{1}^{\star}}\right) \text { on } r=1,
\end{aligned}
$$

where

$$
Z_{h}^{\star}=Z_{h}+\frac{\mathrm{i}}{h \omega} \rho_{0}(h) U_{\theta}^{2}(h) \text { and } Z_{1}^{\star}=Z_{1}-\frac{\mathrm{i}}{\omega} \rho_{0}(1) U_{\theta}^{2}(1)
$$

with the extra terms in $Z_{h}^{\star}$ and $Z_{1}^{\star}$ being centrifugal force due to the swirl.

\subsection{Euler equations for generalised functions}

Next, we consider a time-dependent surface $\mathcal{S}_{f}(t)$, defined by $f_{\mathcal{S}}(\boldsymbol{x}, t)=0$, which separates a fluid into a region where the fluid is moving and a region where it is at rest. The surface $\mathcal{S}_{f}$ represents the blades of a rotor and/or stator, with $f_{\mathcal{S}}(\boldsymbol{x}, t)=0$ corresponding to the blade surfaces. The fluid is at rest inside this surface, and moving outside the surface. Using the convention from Posson and Peake 32 , we choose $f_{\mathcal{S}}$ such that $f_{\mathcal{S}}<0$ when the fluid is at rest and $f_{\mathcal{S}}>0$ when the fluid is moving. We define $\boldsymbol{v}^{\mathcal{S}}$ to be the surface speed, and $\boldsymbol{n}=\boldsymbol{\nabla} f_{\mathcal{S}}(\boldsymbol{x}, t)$ to be the normal. We can then relate the surface speed in the normal direction to the derivative in time, with

$$
\frac{\partial f_{\mathcal{S}}}{\partial t}=-\boldsymbol{v}^{\mathcal{S}} \cdot \boldsymbol{n}
$$

We next consider the Euler equations in Eq. (2) to Eq. (4) in cylindrical coordinates, and then linearise about the base flow. We then perform an exact rearrangement of the Euler equations such that the lefthand side is a linear operator acting on perturbations and the right-hand side consists of all the non-linear effects. Next, we use the theory of generalised functions from Crighton et al. [7, Farassat [1], Grubb [15]. We introduce the velocity, pressure and density distributions, given by $\widetilde{\boldsymbol{u}}=H\left(f_{\mathcal{S}}\right) \boldsymbol{u}, \widetilde{p}=H\left(f_{\mathcal{S}}\right) p$. We multiply the Euler equations by the Heaviside function $H\left(f_{\mathcal{S}}\right)$ and then use the relations from Farassat [1] to relate derivatives of these distributions in terms of source terms and the normal derivative multiplied by the Heaviside function. The differential equations are thus given by

$$
\begin{aligned}
& \frac{\overline{D_{0}} \widetilde{\rho}}{D t}+\widetilde{v} \frac{\mathrm{d} \rho_{0}}{\mathrm{~d} r}+\rho_{0}(\bar{\nabla} \cdot \widetilde{\boldsymbol{u}})=\mathcal{S}_{\text {mass }}+\mathcal{D}_{\text {mass }}, \\
& \rho_{0}\left(\frac{\overline{D_{0}} \widetilde{u}}{D t}+\widetilde{\mathrm{v}} \frac{\mathrm{d} U_{x}}{\mathrm{~d} r}\right)+\frac{\bar{\partial} \widetilde{p}}{\partial x}=\mathcal{S}_{x}+\mathcal{D}_{x} \\
& \rho_{0}\left(\frac{\overline{D_{0}} \widetilde{v}}{D t}-\frac{2 U_{\theta} \widetilde{w}}{r}\right)-\widetilde{\rho} \frac{U_{\theta}^{2}}{r}+\frac{\bar{\partial} \widetilde{p}}{\partial r}=\mathcal{S}_{r}+\mathcal{D}_{r} \\
& \rho_{0}\left(\frac{\overline{D_{0}} \widetilde{w}}{D t}+\frac{\widetilde{v}}{r} \frac{\mathrm{d}}{\mathrm{d} r}\left(r U_{\theta}\right)\right)+\frac{1}{r} \frac{\bar{\partial} \widetilde{p}}{\partial \theta}=\mathcal{S}_{\theta}+\mathcal{D}_{\theta} \\
& \frac{\overline{D_{0}} \widetilde{p}}{D t}+\widetilde{v} \frac{\rho_{0} U_{\theta}^{2}}{r}+\gamma p_{0}(\bar{\nabla} \cdot \widetilde{\boldsymbol{u}})=\mathcal{S}_{e}+\mathcal{D}_{e}
\end{aligned}
$$

where the derivatives with a bar are generalised derivatives, and the material derivative is defined by

$$
\frac{\overline{D_{0}}}{D t}=\frac{\bar{\partial}}{\partial t}+U_{x} \frac{\bar{\partial}}{\partial x}+\frac{U_{\theta}}{r} \frac{\bar{\partial}}{\partial \theta} .
$$


The volume sources terms are defined by

$$
\mathcal{S}_{\text {mass }}=-\bar{\nabla} \cdot(\widetilde{\rho} \widetilde{\boldsymbol{u}}), \quad \mathcal{S}_{e}=-\widetilde{\boldsymbol{u}} \cdot \bar{\nabla} \widetilde{p}-\gamma \tilde{p} \bar{\nabla} \cdot \widetilde{\boldsymbol{u}}
$$

and

$$
\mathcal{S}=\left(\mathcal{S}_{x}, \mathcal{S}_{r}, \mathcal{S}_{\theta}\right)=-\left(\rho_{0}+\widetilde{\rho}\right) \widetilde{\boldsymbol{u}} \cdot \bar{\nabla} \widetilde{\boldsymbol{u}}-\widetilde{\rho} \frac{\overline{D_{0}} \widetilde{\boldsymbol{u}}}{D t}-\widetilde{\rho}\left(\widetilde{v} \frac{\mathrm{d} U_{x}}{\mathrm{~d} r},-\frac{2 U_{\theta} \widetilde{w}}{r}, \frac{\widetilde{v}}{r} \frac{\mathrm{d}}{\mathrm{d} r}\left(r U_{\theta}\right)\right)
$$

The surface source terms are defined by

$$
\begin{gathered}
\mathcal{D}_{\text {mass }}=\delta\left(f_{\mathcal{S}}\right)\left[\rho\left(\underline{\boldsymbol{u}}-\boldsymbol{v}^{\mathcal{S}}\right) \cdot \boldsymbol{n}+\rho_{0}(\boldsymbol{u} \cdot \boldsymbol{n})\right], \\
\mathcal{D}=\left(\mathcal{D}_{x}, \mathcal{D}_{r}, \mathcal{D}_{\theta}\right)=\delta\left(f_{\mathcal{S}}\right)\left[\underline{\rho}\left(\underline{\boldsymbol{u}}-\boldsymbol{v}^{\mathcal{S}}\right) \cdot \boldsymbol{n}+p \boldsymbol{n}\right],
\end{gathered}
$$

and

$$
\mathcal{D}_{e}=\delta\left(f_{\mathcal{S}}\right)\left[p\left(\underline{\boldsymbol{u}}-\boldsymbol{v}^{\mathcal{S}}\right) \cdot \boldsymbol{n}+\gamma p_{0}(\boldsymbol{u} \cdot \boldsymbol{n})+\gamma p(\boldsymbol{u} \cdot \boldsymbol{n})\right],
$$

where $\delta\left(f_{\mathcal{S}}\right)$ is the Dirac delta function.

When the surface $\mathcal{S}_{f}(t)$ is rigid (impermeable and non-vibrating), then $\left(\boldsymbol{u}_{0}+\boldsymbol{u}\right) \cdot \boldsymbol{n}=\boldsymbol{v}^{\mathcal{S}} \cdot \boldsymbol{n}$ and hence we have

$$
\left(\underline{u}-\boldsymbol{v}^{\mathcal{S}}\right) \cdot \boldsymbol{n}=0
$$

so the surface source terms simplify significantly. In addition, if we have no angle of attack on the blades then we also have

$$
\left(\boldsymbol{u}_{0}-\boldsymbol{v}^{\mathcal{S}}\right) \cdot \boldsymbol{n}=0
$$

which further simplifies the source terms. The surface source term $\mathcal{D}$ is related to the loading noise and $\mathcal{D}_{\text {mass }}$ is related to thickness noise from the surfaces 32 .

\subsection{Derivation of acoustic analogy}

Next, we combine Eq. (21) to Eq. 25) into a single equation for the pressure perturbation, using a similar method to Posson and Peake 32 . We first note that the material derivative commutes with circumferential and axial derivatives but not radial derivatives, with

$$
\frac{\bar{\partial}}{\partial r}\left(\frac{\overline{D_{0}}}{D t}\right)=\frac{\overline{D_{0}}}{\overline{D t}} \frac{\bar{\partial}}{\partial r}+U_{x}^{\prime}(r) \frac{\bar{\partial}}{\partial x}+\left(\frac{U_{\theta}(r)}{r}\right)^{\prime} \frac{\bar{\partial}}{\partial \theta} .
$$

Using Eq. (24) we express the material derivative of $\widetilde{w}$ in terms of $\widetilde{v}, \widetilde{p}$ and source terms:

$$
\rho_{0} \frac{\overline{D_{0}} \widetilde{w}}{D t}=\mathcal{D}_{\theta}+\mathcal{S}_{\theta}-\frac{1}{r} \frac{\bar{\partial} \widetilde{p}}{\partial \theta}-\widetilde{v} \frac{\rho_{0}}{r} \frac{\mathrm{d}}{\mathrm{d} r}\left(r U_{\theta}\right) .
$$

Combining Eq. 21) and Eq. 25 allows us to express the material derivative of the density $\widetilde{\rho}$ similarly:

$$
\frac{\overline{D_{0}} \widetilde{\rho}}{D t}=\mathcal{D}_{\text {mass }}+\mathcal{S}_{\text {mass }}-\frac{\mathcal{D}_{e}+\mathcal{S}_{e}}{c_{0}^{2}}+\frac{1}{c_{0}^{2}} \frac{\overline{D_{0}} \widetilde{p}}{D t}+\frac{\rho_{0} U_{\theta}^{2}}{r c_{0}^{2}} \widetilde{v}-\rho_{0}^{\prime} \widetilde{v}
$$

Next, we take the material derivative of Eq. (23) and substitute in Eq. (35) and Eq. (36). We find

$$
\rho_{0} \mathcal{R}(\widetilde{v})=\mathcal{T}(\widetilde{p})+\mathbb{S}_{1}^{M},
$$

where the differential operators $\mathcal{R}$ and $\mathcal{T}$ are defined by

$$
\mathcal{R}=\frac{{\overline{D_{0}}}^{2}}{D t^{2}}+\mathcal{U}_{\theta} \text { and } \mathcal{T}=-\frac{\overline{D_{0}}}{D t} \frac{\bar{\partial}}{\partial r}-\frac{2 U_{\theta}}{r^{2}} \frac{\bar{\partial}}{\partial \theta}+\frac{U_{\theta}^{2}}{r c_{0}^{2}} \frac{\overline{D_{0}}}{D t},
$$


with

$$
\mathcal{U}_{\theta}=\frac{2 U_{\theta}}{r^{2}} \frac{\mathrm{d}}{\mathrm{d} r}\left(r U_{\theta}\right)+\frac{U_{\theta}^{2}}{r}\left(\frac{\rho_{0}^{\prime}}{\rho_{0}}-\frac{U_{\theta}^{2}}{r c_{0}^{2}}\right)
$$

and the source term $\mathbb{S}_{1}^{M}$ is given by

$$
\mathbb{S}_{1}^{M}=\frac{\overline{D_{0}}}{D t}\left(\mathcal{D}_{r}+\mathcal{S}_{r}\right)+\frac{U_{\theta}^{2}}{r}\left(\mathcal{D}_{\text {mass }}+\mathcal{S}_{\text {mass }}-\frac{\mathcal{D}_{e}+\mathcal{S}_{e}}{c_{0}^{2}}\right)+\frac{2 U_{\theta}}{r}\left(\mathcal{D}_{\theta}+\mathcal{S}_{\theta}\right) .
$$

Compared to the notation in Posson and Peake [32] we have $\mathcal{R}=-\mathcal{D}$ and $\mathcal{T}=-\mathcal{M}$. To simplify matters slightly in what follows we introduce $\mathbb{U}_{\theta}(r)=\left(r U_{\theta}\right)^{\prime} / r^{2}$. The next step is to differentiate Eq. 22 with respect to $x$, and differentiate Eq. (24) with respect to $\theta$ and divide by $r$, which gives

$$
\rho_{0} \frac{\overline{D_{0}}}{\overline{D t}} \frac{\bar{\partial} \widetilde{u}}{\partial x}=-\rho_{0} U_{x}^{\prime} \frac{\bar{\partial} \widetilde{v}}{\partial x}-\frac{\bar{\partial}^{2} \widetilde{p}}{\partial x^{2}}+\frac{\bar{\partial}}{\partial x}\left(\mathcal{D}_{x}+\mathcal{S}_{x}\right), \quad \rho_{0} \frac{\overline{D_{0}}}{D t}\left(\frac{1}{r} \frac{\bar{\partial} \widetilde{w}}{\partial \theta}\right)=-\rho_{0} \mathbb{U}_{\theta} \frac{\bar{\partial} \widetilde{v}}{\partial \theta}-\frac{1}{r^{2}} \frac{\bar{\partial}^{2} \widetilde{p}}{\partial \theta^{2}}+\frac{1}{r} \frac{\bar{\partial}}{\partial \theta}\left(\mathcal{D}_{\theta}+\mathcal{S}_{\theta}\right) .
$$

We then divide Eq. 25) by $c_{0}^{2}(r)$ and then take the material derivative of it, which gives

$$
\frac{1}{c_{0}^{2}} \frac{{\overline{D_{0}}}^{2} \widetilde{p}}{D t^{2}}+\rho_{0} \frac{\overline{D_{0}}}{D t} \frac{\bar{\partial} \widetilde{u}}{\partial x}+\rho_{0} \frac{\overline{D_{0}}}{D t}\left(\frac{\bar{\partial} \widetilde{v}}{\partial r}+\frac{\widetilde{v}}{r}\right)+\rho_{0} \frac{\overline{D_{0}}}{D t}\left(\frac{1}{r} \frac{\bar{\partial} \widetilde{w}}{\partial \theta}\right)+\frac{\rho_{0} U_{\theta}^{2}}{r c_{0}^{2}} \frac{\overline{D_{0}} \widetilde{v}}{D t}=\frac{\overline{D_{0}}}{D t}\left(\frac{\mathcal{D}_{e}+\mathcal{S}_{e}}{c_{0}^{2}}\right) .
$$

Substituting the expressions from Eq. 41) into Eq. 42, then gives

$$
\left(\frac{1}{c_{0}^{2}} \frac{{\overline{D_{0}}}^{2}}{D t^{2}}-\frac{\bar{\partial}^{2}}{\partial x^{2}}-\frac{1}{r^{2}} \frac{\bar{\partial}^{2}}{\partial \theta^{2}}\right) \widetilde{p}+\rho_{0} \frac{\overline{D_{0}}}{D t} \frac{\bar{\partial} \widetilde{v}}{\partial r}+\rho_{0}\left(\frac{1}{r} \frac{\overline{D_{0}}}{\overline{D t}}-U_{x}^{\prime} \frac{\bar{\partial}}{\partial x}-\mathbb{U}_{\theta} \frac{\bar{\partial}}{\partial \theta}+\frac{U_{\theta}^{2}}{r c_{0}^{2}} \frac{\overline{D_{0}}}{D t}\right) \widetilde{v}=\mathbb{S}_{2}^{M},
$$

where the source term $\mathbb{S}_{2}^{M}$ is given by

$$
\mathbb{S}_{2}^{M}=\frac{\overline{D_{0}}}{D t}\left(\frac{\mathcal{D}_{e}+\mathcal{S}_{e}}{c_{0}^{2}}\right)-\frac{\bar{\partial}}{\partial x}\left(\mathcal{S}_{x}+\mathcal{D}_{x}\right)-\frac{1}{r} \frac{\bar{\partial}}{\partial \theta}\left(\mathcal{S}_{\theta}+\mathcal{D}_{\theta}\right)
$$

In Eq. 43 we have eliminated the axial and circumferential velocities $\widetilde{u}$ and $\widetilde{w}$ from the left-hand side, as well as the density $\widetilde{\rho}$.

The final step in the derivation of the acoustic analogy is to eliminate the radial velocity $\widetilde{v}$ from the left-hand side of Eq. (43). To do this we want to insert equation Eq. (37) into Eq. (43), but first we need to ensure all the terms involving $\widetilde{v}$ in Eq. 43 are of the form $\rho_{0} \mathcal{R}(\widetilde{v})$. The operator $\mathcal{R}$ commutes with derivatives with respect to $\theta$ and $x$, as well as the material derivative, but not derivatives with respect to $r$. We calculate that

$$
\mathcal{R}\left(\frac{\overline{D_{0}}}{D t} \frac{\bar{\partial} \widetilde{v}}{\partial r}\right)==\frac{\overline{D_{0}}}{D t} \frac{\bar{\partial}}{\partial r} \mathcal{R}(v)-2 U_{x}^{\prime} \frac{\bar{\partial}}{\partial x} \frac{{\overline{D_{0}}}^{2} \widetilde{v}}{D t^{2}}-2\left(\frac{U_{\theta}}{r}\right)^{\prime} \frac{\bar{\partial}}{\partial \theta} \frac{{\overline{D_{0}}}^{2} \widetilde{v}}{D t^{2}}-\mathcal{U}_{\theta}^{\prime} \frac{\overline{D_{0}} \widetilde{v}}{D t}
$$

If we apply the differential operator $\mathcal{R}$ to Eq. 43, and then insert Eq. 45, we get

$$
\begin{aligned}
&\left(\frac{1}{c_{0}^{2}} \frac{{\overline{D_{0}}}^{2}}{D t^{2}}-\frac{\bar{\partial}^{2}}{\partial x^{2}}-\frac{1}{r^{2}}\right.\left.\frac{\bar{\partial}^{2}}{\partial \theta^{2}}\right) \mathcal{R}(\widetilde{p})+\rho_{0}\left(\frac{1}{r} \frac{\overline{D_{0}}}{D t}-U_{x}^{\prime} \frac{\bar{\partial}}{\partial x}-\mathbb{U}_{\theta} \frac{\bar{\partial}}{\partial \theta}+\left(\frac{U_{\theta}^{2}}{r c_{0}^{2}}-\frac{\rho_{0}^{\prime}}{\rho_{0}}\right) \frac{\overline{D_{0}}}{D t}\right) \mathcal{R}(\widetilde{v}) \\
&+\frac{\overline{D_{0}}}{D t} \frac{\bar{\partial}}{\partial r}\left(\rho_{0} \mathcal{R}(v)\right)-\rho_{0}\left[2 U_{x}^{\prime} \frac{\bar{\partial}}{\partial x} \frac{{\overline{D_{0}}}^{2} \widetilde{v}}{D t^{2}}+2\left(\frac{U_{\theta}}{r}\right)^{\prime} \frac{\bar{\partial}}{\partial \theta} \frac{{\overline{D_{0}}}^{2} \widetilde{v}}{D t^{2}}+\mathcal{U}_{\theta}^{\prime} \frac{\overline{D_{0}} \widetilde{v}}{D t}\right]=\mathcal{R}\left(\mathbb{S}_{2}^{M}\right)
\end{aligned}
$$

Despite applying $\mathcal{R}$ to Eq. (43) once, we still have some $\widetilde{v}$ terms which are not of the form $\rho_{0} \mathcal{R}(\widetilde{v})$. However, these terms now only involve material derivatives and derivatives with respect to $\theta$ and $x$, so we just apply 
the differential operator $\mathcal{R}$ to Eq. (46) once more. Finally, we use Eq. (37) to eliminate velocity terms, which gives

$$
\mathcal{F}^{M}(\widetilde{p})=\mathbb{S}^{M}
$$

The operator $\mathcal{F}^{M}$ is defined by

$$
\begin{aligned}
& \mathcal{F}^{M}:=\left(\frac{1}{c_{0}^{2}} \frac{{\overline{D_{0}}}^{2}}{D t^{2}}-\frac{\bar{\partial}^{2}}{\partial x^{2}}-\frac{1}{r^{2}} \frac{\bar{\partial}^{2}}{\partial \theta^{2}}\right) \mathcal{R}^{2}+\left(\frac{1}{r} \frac{\overline{D_{0}}}{D t}-U_{x}^{\prime} \frac{\bar{\partial}}{\partial x}-\mathbb{U}_{\theta} \frac{\bar{\partial}}{\partial \theta}+\left(\frac{U_{\theta}^{2}}{r c_{0}^{2}}-\frac{\rho_{0}^{\prime}}{\rho_{0}}\right) \overline{\frac{D_{0}}{D t}}\right) \mathcal{R} \mathcal{T} \\
& +\mathcal{R} \frac{\overline{D_{0}}}{D t} \frac{\bar{\partial}}{\partial r} \mathcal{T}-\frac{\overline{D_{0}}}{D t}\left[2 U_{x}^{\prime} \frac{\bar{\partial}}{\partial x} \frac{\overline{D_{0}}}{\overline{D t}}+2\left(\frac{U_{\theta}}{r}\right)^{\prime} \frac{\bar{\partial}}{\partial \theta} \frac{\overline{D_{0}}}{D t}+\mathcal{U}_{\theta}^{\prime}\right] \mathcal{T},
\end{aligned}
$$

and the source term is

$$
\begin{aligned}
\mathbb{S}^{M}=\mathcal{R}^{2}\left(\mathbb{S}_{2}^{M}\right)-\mathcal{R}\left(\frac{\overline{D_{0}}}{\overline{D t}}\left(\frac{1}{r}+\frac{\bar{\partial}}{\partial r}\right)-U_{x}^{\prime} \frac{\bar{\partial}}{\partial x}-\mathbb{U}_{\theta} \frac{\bar{\partial}}{\partial \theta}+\left(\frac{U_{\theta}^{2}}{r c_{0}^{2}}-\frac{\rho_{0}^{\prime}}{\rho_{0}}\right) \frac{\overline{D_{0}}}{\overline{D t}}\right) \mathbb{S}_{1}^{M} \\
+\frac{\overline{D_{0}}}{D t}\left[2 U_{x}^{\prime} \frac{\bar{\partial}}{\partial x} \frac{\overline{D_{0}}}{D t}+2\left(\frac{U_{\theta}}{r}\right)^{\prime} \frac{\bar{\partial}}{\partial \theta} \frac{\overline{D_{0}}}{D t}+\mathcal{U}_{\theta}^{\prime}\right] \mathbb{S}_{1}^{M} .
\end{aligned}
$$

\subsection{Reducing to the form in Posson and Peake [32]}

When the entropy is constant, we calculate that

$$
\frac{\rho_{0}^{\prime}(r)}{\rho_{0}(r)}=\frac{U_{\theta}^{2}(r)}{r c_{0}^{2}(r)} .
$$

Thus, we find for constant entropy

$$
\mathcal{F}^{M}=\mathcal{F}^{P P},
$$

where $\mathcal{F}^{P P}$ is the sixth order differential operator from Posson and Peake [32].

For the source terms, we can see that comparing Eq. (49) with Posson and Peake [32, Equation 3.7], then we will get exact agreement provided that

$$
\mathbb{S}_{1}^{M}=\mathbb{S}_{1}^{P P}+\mathbb{S}_{F W H, 1}^{P P} \text { and } \mathbb{S}_{2}^{M}=\mathbb{S}_{2}^{P P}+\mathbb{S}_{F W H, 2}^{P P},
$$

where the source terms with a ${ }^{P P}$ superscript are defined in Posson and Peake [32, Equation 3.9,3.10]. We show that this holds exactly in the case of constant entropy in Appendix B.

\section{Asymptotic and numerical Green's function}

Even though the base flow entropy now varies, we can still use a very similar method to Mathews and Peake [26 to find the eigenmodes and Green's function of the swirling flow. When we consider the highfrequency limit $(\omega \rightarrow \infty)$, the differential equation and boundary conditions are unchanged from Mathews and Peake [26, but the base flow speed of sound, density and pressure vary. For the numerical method, we now get a fifth equation to solve in our eigenvalue problem, but otherwise the method is the same. We briefly summarise the methods below.

We first consider the modified Green's function $\widehat{p}_{\omega}$ of $\mathcal{F}^{M}$, which satisfies

$$
\mathcal{F}^{M}\left(\widehat{p}_{\omega} e^{-\mathrm{i} \omega t}\right)=\frac{1}{2 \pi} \frac{D_{0}^{2}}{D t^{2}} \mathcal{R}\left(\delta\left(\boldsymbol{x}-\boldsymbol{x}_{0}\right) e^{-\mathrm{i} \omega t}\right) .
$$

We consider the Green's function $\widehat{p}_{\omega}$ rather than a standard Green's function since it would be more useful were we to calculate the pressure from source terms (using the method from Posson and Peake [32] but extended for a isentropic flow). If we were to solve for the standard Greens function instead of the reduced 
Greens function, the method would be exactly the same as what follows, as was the case for constant entropy in Mathews and Peake [26]. We look for a Green's function of the form

$$
\widehat{p}_{\omega}\left(\boldsymbol{x} \mid \boldsymbol{x}_{0}\right)=\frac{1}{4 \pi^{2}} \sum_{n=-\infty}^{\infty} e^{\mathrm{i} n\left(\theta-\theta_{0}\right)} \int_{\mathbb{R}} \widehat{p}_{n}\left(r \mid r_{0} ; \omega, k\right) e^{\mathrm{i} k\left(x-x_{0}\right)} \mathrm{d} k
$$

where we choose a circumferential Fourier series to ensure periodicity. Using standard results from Bender and Orszag [1] gives $\widehat{p}_{n}$ as

$$
\widehat{p}_{n}\left(r \mid r_{0} ; \omega, k\right)=\frac{1}{2 \pi r_{0} \mathcal{W}\left(r_{0}, k\right)}\left\{\begin{array}{ll}
g_{1}\left(r_{0} ; k\right) g_{2}(r ; k) & r \leq r_{0} \\
g_{2}\left(r_{0} ; k\right) g_{1}(r ; k) & r>r_{0}
\end{array},\right.
$$

where $\mathcal{W}\left(r_{0}, k\right)$ is the Wrońskian of $g_{1}(r ; k)$ and $g_{2}(r ; k)$. The functions $g_{1}(r ; k)$ and $g_{2}(r ; k)$ solve the second order ordinary differential equation

$$
\begin{array}{r}
\frac{p_{0}}{c_{0}^{2} r}\left(\mathcal{U}_{\theta}-\Omega^{2}\right)^{2} \Omega^{2} \frac{\mathrm{d}}{\mathrm{d} r}\left(\frac{r c_{0}^{2}}{p_{0}\left(\Omega^{2}-\mathcal{U}_{\theta}\right)} \frac{\mathrm{d} g_{j}}{\mathrm{~d} r}\right)+\left[\left(\mathcal{U}_{\theta}-\Omega^{2}\right)^{2}\left(\frac{\Omega^{2}}{c_{0}^{2}}-k^{2}-\frac{n^{2}}{r^{2}}\right)\right. \\
\left.+\Upsilon\left(\mathcal{U}_{\theta}-\Omega^{2}\right)\left[\Upsilon+\Omega\left(\frac{1}{r}-\frac{\rho_{0}^{\prime}}{\rho_{0}}\right)\right]-\Upsilon^{\prime} \Omega\left(\Omega^{2}-\mathcal{U}_{\theta}\right)+\Upsilon\left[\Omega\left(\Omega^{2}-\mathcal{U}_{\theta}\right)\right]^{\prime}\right] g_{j}=0
\end{array}
$$

with $g_{1}(r ; k)$ satisfying the (Fourier transformed) Myers boundary condition at $r=1$ and $g_{2}(r ; k)$ satisfying the Myers boundary condition at $r=h$. The functions $\Omega(r, k)$ and $\Upsilon(r, k)$ are given by

$$
\Omega(r, k)=\omega-k U_{x}(r)-\frac{n U_{\theta}(r)}{r} \text { and } \Upsilon(r, k)=\frac{U_{\theta}^{2}(r)}{r c_{0}^{2}(r)} \Omega(r, k)+\frac{2 n U_{\theta}(r)}{r^{2}} .
$$

\subsection{Numerical eigenmodes}

To calculate the eigenmodes numerically, we first take the linearised mass, momentum and energy equations (in terms of the entropy), which gives us five equations. Using the constitutive relations for a perfect gas, Eq. (5) and Eq. (6), we can eliminate the density perturbation using (to first order)

$$
\rho=\frac{p}{c_{0}^{2}}-\frac{\rho_{0} s}{c_{p}}
$$

We then Fourier transform the perturbations $u, v, w, p$ and $s$ in the frequency and axial domains, and Fourier series in the circumferential direction, which we denote by the respective capital letters. We then solve the resulting eigenvalue problem for the axial wavenumber $k$. After rearranging to make the right-hand side simpler, the final eigenvalue problem is given by

$$
\begin{array}{r}
-\frac{U_{x} \bar{\Omega}}{c_{0}^{2} \zeta} \mathrm{i} U+\left[\frac{U_{x}}{c_{0}^{2} \zeta} \frac{\mathrm{d} U_{x}}{\mathrm{~d} r}-\frac{1}{r \zeta}-\frac{U_{\theta}^{2}}{\zeta r c_{0}^{2}}\right] V-\frac{1}{\zeta} \frac{\mathrm{d} V}{\mathrm{~d} r}-\frac{n}{r \zeta} \mathrm{i} W+\frac{\mathrm{i} \bar{\Omega}}{c_{0}^{2} \rho_{0} \zeta} P=k \mathrm{i} U, \\
\frac{\bar{\Omega}}{U_{x}} V-\frac{2 U_{\theta}}{r U_{x}} \mathrm{i} W+\frac{\mathrm{i}}{\rho_{0} U_{x}} \frac{\mathrm{d} P}{\mathrm{~d} r}-\frac{\mathrm{i} U_{\theta}^{2}}{\rho_{0} U_{x} r c_{0}^{2}} P+\mathrm{i} \frac{U_{\theta}^{2}}{r c_{p} U_{x}} S=k V, \\
-\frac{1}{U_{x}}\left[\frac{U_{\theta}}{r}+\frac{\mathrm{d} U_{\theta}}{\mathrm{d} r}\right] V+\frac{\bar{\Omega}}{U_{x}} \mathrm{i} W-\frac{\mathrm{i} n}{r \rho_{0} U_{x}} P=k \mathrm{i} W, \\
\frac{\rho_{0} \bar{\Omega}}{\zeta} U+\mathrm{i} \frac{\rho_{0}}{\zeta}\left[\frac{\mathrm{d} U_{x}}{\mathrm{~d} r}-\left(1+\frac{U_{\theta}^{2}}{c_{0}^{2}}\right) \frac{U_{x}}{r}\right] V-\frac{\mathrm{i} \rho_{0} U_{x}}{\zeta} \frac{\mathrm{d} V}{\mathrm{~d} r}-\frac{\mathrm{i} n \rho_{0} U_{x}}{r \zeta} \mathrm{i} W-\frac{U_{x} \bar{\Omega}}{c_{0}^{2} \zeta} P=k P, \\
\mathrm{i} \frac{1}{U_{x}} \frac{\mathrm{d} s_{0}}{\mathrm{~d} r} V+\frac{\bar{\Omega}}{U_{x}} S=k S,
\end{array}
$$


where

$$
\bar{\Omega}=\omega-\frac{n U_{\theta}}{r} \text { and } \zeta=1-\frac{U_{x}^{2}}{c_{0}^{2}} .
$$

The boundary conditions for the eigenvalue problem are calculated by Fourier transforming the standard Myers boundary conditions in Eq. (17), and are

$$
Z_{h} \frac{\omega V(h)}{U_{x}(h)}+\frac{\bar{\Omega}(h) P(h)}{U_{x}(h)}-k P(h)=0, \quad Z_{1} \frac{\omega V(1)}{U_{x}(1)}-\frac{\bar{\Omega}(1) P(1)}{U_{x}(1)}+k P(1)=0 .
$$

We solve this eigenvalue problem using Chebfun 9 in MATLAB, which avoids having to discretise the derivatives. To remove the eigenvalue from the boundary condition we introduce a sixth variable $\bar{P}=k P$. We validated the solver by checking that when the entropy is constant we recover the results from [26] and 32 .

A homentropic fluid has constant entropy, so $s_{0}(r)$ is constant. As a result, the only possible non-zero solution $S$ to Eq. (63) is when $k$ is in the critical layer, which is defined by

$$
\inf _{h<r<1} \frac{\bar{\Omega}}{U_{x}} \leq k \leq \sup _{h<r<1} \frac{\bar{\Omega}}{U_{x}} .
$$

So, away from the critical layer (and hence for all acoustic eigenmodes), $S=0$ and we only need to solve a system of four differential equations. These are the four equations that are solved in Posson and Peake [32] and Mathews and Peake [26].

\subsection{Asymptotic eigenmodes}

We consider the differential equation in the high-frequency limit, which allows us to use the WKB method as in Mathews and Peake 26. We first make the change of variables

$$
g_{j}(r ; \kappa)=\frac{\rho_{0}^{1 / 2}(r) \Phi(r, \kappa) \mathcal{P}_{j}(r ; \kappa)}{r^{1 / 2}},
$$

where $k=\omega \kappa$, so that the leading order behaviour of the differential equation is given by

$$
\mathcal{P}_{j}^{\prime \prime}(r ; \kappa)+\omega^{2} q_{n}(r, \kappa) \mathcal{P}_{j}(r ; \kappa)=0 \text { with } q_{n}(r, \kappa)=\left(\frac{\Phi^{2}(r, \kappa)}{c_{0}^{2}(r)}-\kappa^{2}-\frac{\eta^{2}}{r^{2}}\right),
$$

with $n=\omega \eta$ and $\Omega=\omega \Phi$. The boundary conditions become

$$
\frac{\mathrm{d} \mathcal{P}_{2}}{\mathrm{~d} r}(h ; \kappa)+f_{2}(h, \kappa) \mathcal{P}_{2}(h ; \kappa)=0 \text { and } \frac{\mathrm{d} \mathcal{P}_{1}}{\mathrm{~d} r}(1 ; \kappa)+f_{1}(1, \kappa) \mathcal{P}_{1}(1 ; \kappa)=0,
$$

where $f_{1}(1, \kappa)$ and $f_{2}(h, \kappa)$ can easily be determined from the Fourier transformed Myers boundary conditions as

$$
f_{2}(h, \kappa)=\frac{\mathrm{i} \rho_{0}(h) \Phi^{2}(h, \kappa)}{Z_{h}} \omega+\frac{1}{2}\left(2 \frac{\frac{\partial}{\partial r} \Phi(h, \kappa)}{\Phi(h, \kappa)}+\frac{\rho_{0}^{\prime}(h)}{\rho_{0}(h)}-\frac{1}{h}\right)-\frac{\Upsilon(h)}{\omega \Phi(h, \kappa)}-\frac{\mathrm{i} \rho_{0}(h) \mathcal{U}_{\theta}(h)}{\omega Z_{h}},
$$

and

$$
f_{1}(1, \kappa)=-\frac{\mathrm{i} \rho_{0}(1) \Phi^{2}(1, \kappa)}{Z_{1}} \omega+\frac{1}{2}\left(2 \frac{\frac{\partial}{\partial r} \Phi(1, \kappa)}{\Phi(1, \kappa)}+\frac{\rho_{0}^{\prime}(1)}{\rho_{0}(1)}-1\right)-\frac{\Upsilon(1)}{\omega \Phi(1, \kappa)}+\frac{\mathrm{i} \rho_{0}(1) \mathcal{U}_{\theta}(1)}{\omega Z_{1}} .
$$

Under our change of variables, the Green's function $\widehat{p}_{n}$ is now given by

$$
\widehat{p}_{n}\left(r \mid r_{0} ; \kappa\right)=\left(\frac{\rho_{0}(r) r_{0}}{\rho_{0}\left(r_{0}\right) r}\right)^{1 / 2} \frac{\Phi(r, \kappa)}{2 \pi r_{0} \mathcal{V}\left(r_{0}, \kappa\right) \Phi\left(r_{0}, \kappa\right)}\left\{\begin{array}{ll}
\mathcal{P}_{1}\left(r_{0} ; \kappa\right) \mathcal{P}_{2}(r ; \kappa) & r \leq r_{0} \\
\mathcal{P}_{2}\left(r_{0} ; \kappa\right) \mathcal{P}_{1}(r ; \kappa) & r>r_{0}
\end{array},\right.
$$


(a)

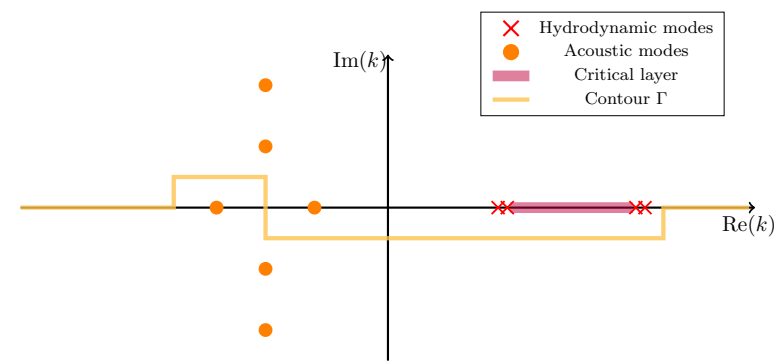

(b)

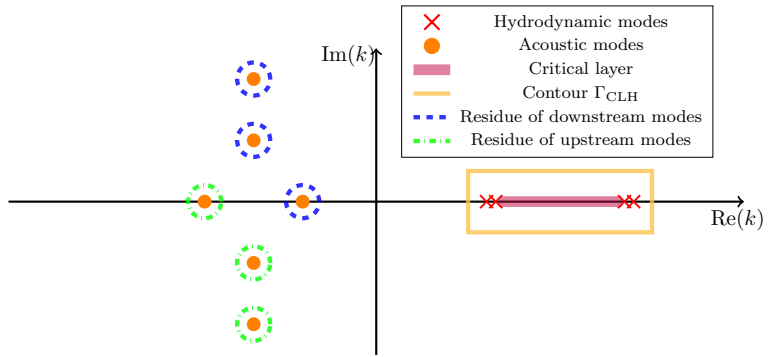

Figure 3: Schematic of eigenmodes and integration contour. (a) Original contour $\Gamma$; (b) contour $\Gamma_{\mathrm{CLH}}$ and residues. We also see acoustic modes (orange circles), hydrodynamic modes (red crosses), critical layer (solid pink line), residues of the downstream eigenmodes (blue, dashed) and residue of the upstream eigenmodes (green, dot-dashed).

where the Wrońskian $\mathcal{V}(r, \kappa)$ of $\mathcal{P}_{1}(r ; \kappa)$ and $\mathcal{P}_{2}(r ; \kappa)$ is now independent of $r$ by Abel's theorem [2]. We next calculate that $\mathcal{P}_{j}(r ; \kappa)$ is given by

$$
\mathcal{P}_{j}(r ; \kappa)=A^{j}(\kappa) \mathscr{A}(r, \kappa)+B^{j}(\kappa) \mathscr{B}(r, \kappa)
$$

and

$$
\frac{\mathrm{d} \mathcal{P}_{j}}{\mathrm{~d} r}(r ; \kappa)=A^{j}(\kappa) \mathbb{A}(r, \kappa)+B^{j}(\kappa) \mathbb{B}(r, \kappa),
$$

where $\mathscr{A}(r, \kappa), \mathscr{B}(r, \kappa), \mathbb{A}(r, \kappa)$ and $\mathbb{B}(r, \kappa)$, the linearly independent solutions and their derivatives, are defined in Appendix A. Setting $A^{j}(\kappa)=1$ and applying the boundary conditions in Eq. 69 gives

$$
B^{2}(\kappa)\left(\mathbb{B}(h, \kappa)+f_{2}(h, \kappa) \mathscr{B}(h, \kappa)\right)=-\mathbb{A}(h, \kappa)-f_{2}(h, \kappa) \mathscr{A}(h, \kappa),
$$

and

$$
B^{1}(\kappa)\left(\mathbb{B}(1, \kappa)+f_{1}(1, \kappa) \mathscr{B}(1, \kappa)\right)=-\mathbb{A}(1, \kappa)-f_{1}(1, \kappa) \mathscr{A}(1, \kappa),
$$

which we solve to find $B^{1}(\kappa)$ and $B^{2}(\kappa)$. We then calculate that the Wrońskian is given by $\mathcal{V}(\kappa)=$ $\omega\left[B^{1}(\kappa)-B^{2}(\kappa)\right]$, so the dispersion relation is given by

$$
\mathcal{V}(\kappa)=\omega\left[B^{1}(\kappa)-B^{2}(\kappa)\right]=0
$$

We solve this numerically (by using the rootfinding algorithm fsolve in MATLAB with the numerical eigenmode as a starting guess) to find the asymptotic eigenmodes.

\subsection{Asymptotic Green's function}

To calculate $\widehat{p}_{\omega}$ we find the inverse Fourier transform of $\widehat{p}_{n}$, but instead of using the real line we use the contour $\Gamma$ given in Figure 3a, which was determined by considering the standard causal contour. To perform the integration we close the contour in the upper or lower half plane. When $x>x_{0}$ we close the contour in the upper half plane, and the Green's function is equal to the sum of the residues at the downstream acoustic eigenmodes, plus a critical layer contribution. When $x<x_{0}$ we close the contour in the lower half plane, and get a sum of the residues at the upstream acoustic eigenmodes, with no contribution from the critical layer.

\subsubsection{Contribution from acoustic eigenmodes}

The total contribution from the acoustic eigenmodes is given by

$$
\widehat{p}_{\omega}^{A}\left(\boldsymbol{x} \mid \boldsymbol{x}_{0}\right)=\sum_{n=-\infty}^{\infty} e^{\mathrm{i} n\left(\theta-\theta_{0}\right)} \sum_{\mathcal{K}_{n}^{ \pm}} \widehat{p}_{n}^{m}\left(x, r \mid x_{0}, r_{0}\right),
$$


where $\mathcal{K}_{n}^{+}$consists of all downstream acoustic modes, $\mathcal{K}_{n}^{-}$consists of all upstream acoustic modes and $\widehat{p}_{n}^{m}$ is the residue of $\widehat{p}_{n}$ at the $m$-th mode in $\mathcal{K}_{n}^{ \pm}$. We calculate that

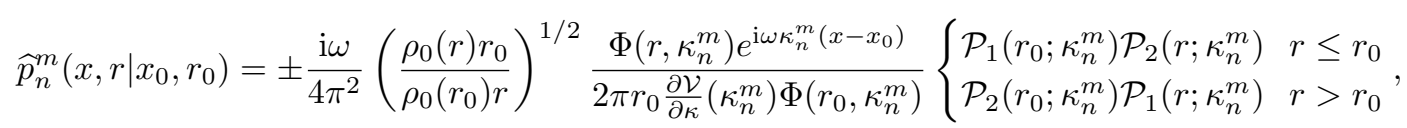

with the \pm coming from the sign of $x-x_{0}$. We let $\widehat{p}_{n}^{A}\left(x, r \mid x_{0}, r_{0}\right)=\sum \widehat{p}_{n}^{m}$ be the total acoustic contribution at each azimuthal number, which is calculated by summing over all upstream or downstream modes. The derivative of the Wrońskian with respect to $\kappa$ can be analytically calculated, although the exact form would be quite complicated.

\subsubsection{Contribution from critical layer}

We calculate the contribution from the critical layer by using a counter clockwise contour $\Gamma_{\mathrm{CLH}}$ enclosing the critical layer and hydrodynamic modes, which is shown in Figure 3. This is very expensive to calculate numerically, but quite inaccurate to calculate asymptotically using the method in Mathews [25]. It was shown in Posson and Peake 32 that the contribution from the critical layer is small enough to ignore except in a handful of cases, so we will ignore it for the rest of this discussion.

\subsection{Numerical Green's function}

For the numerical method we only consider the acoustic Green's function, and ignore the critical layer contribution. Once we have calculated the eigenmodes (and eigenfunctions) for the Green's function we can use a similar formula to Eq. $\sqrt{79}$ to calculate the contribution from each mode, with the only remaining difficulty calculating the derivative of the Wrońskian with respect to $k$. The method used to do this is given in Posson and Peake [32] and Mathews and Peake [26], and the isentropic flow has no effect on the method.

\section{Effect of entropy on acoustic modes}

We will mainly consider the base flow entropy $s_{0}(r)=-\log \left(r^{\beta}\right)$, where we will vary the parameter $\beta$. When we vary this base flow entropy, there are three main features. First, as we increase the entropy gradient the line of cut-off modes shifts right in the complex plan. Second, as we increase the entropy gradient we reduce the number of cut-on modes. Third, when the duct has lining we find both upstream and downstream surface modes, once the entropy parameter $\beta$ is below a certain threshold. The first two effects are universal for any swirling flow, frequency or azimuthal number (but same entropy profile $\left.s_{0}(r)=-\log \left(r^{\beta}\right)\right)$. The third effect has a more complicated dependence on these parameters, and would require the use of a similar method to Rienstra [34] or Brambley and Peake [3] but extended to isentropic, swirling flow to fully determine the effect of the entropy gradient.

We can easily explain the first two effects by analogy with uniform axial flow in a hard-walled duct. As we increase the entropy $s_{0}(r)$, we increase the speed of sound $c_{0}(r)$. This therefore reduces the Mach number $M(r)$ of the flow. In uniform flow, the line of cut-off modes is given by $\operatorname{Re}(k)=-\omega M /\left(1-M^{2}\right)$. Thus, increasing the entropy gradient causes the line of cut-off modes to shift right. Furthermore, the condition for cut-on modes in a duct is $\alpha^{2}<\omega /\left(1-M^{2}\right)$, with $\alpha$ given in Vilenski and Rienstra 38] and involves finding zeros of Bessel functions. As we increase the entropy gradient, we decrease $M$ and hence decrease $\omega /\left(1-M^{2}\right)$. Thus, we get less cut-on modes since previously accepted values of $\alpha$ no longer satisfy the cut-on condition.

For other choices of base flow entropy, the speed of sound has a more complicated form, as given in Eq. 10. and thus it is unclear whether increasing the entropy will always lead to an increase in speed of sound and hence less cut-on modes and cut-off modes shifting right.

We consider two different swirling flows in a lined duct of impedance $Z=1-2 \mathrm{i}$. In Figure 4 we consider uniform axial flow and zero swirl, while in Figure 5 we consider polynomial axial and swirling flow of the form $U_{x}(r)=0.3+0.2 r^{2}$ and $U_{\theta}(r)=0.2 r+0.1 / r$. We plot the numerical eigenmodes in Figures 4 and 5 and give a table comparing a selection of the numerical and asymptotic eigenmodes. We observe that both 


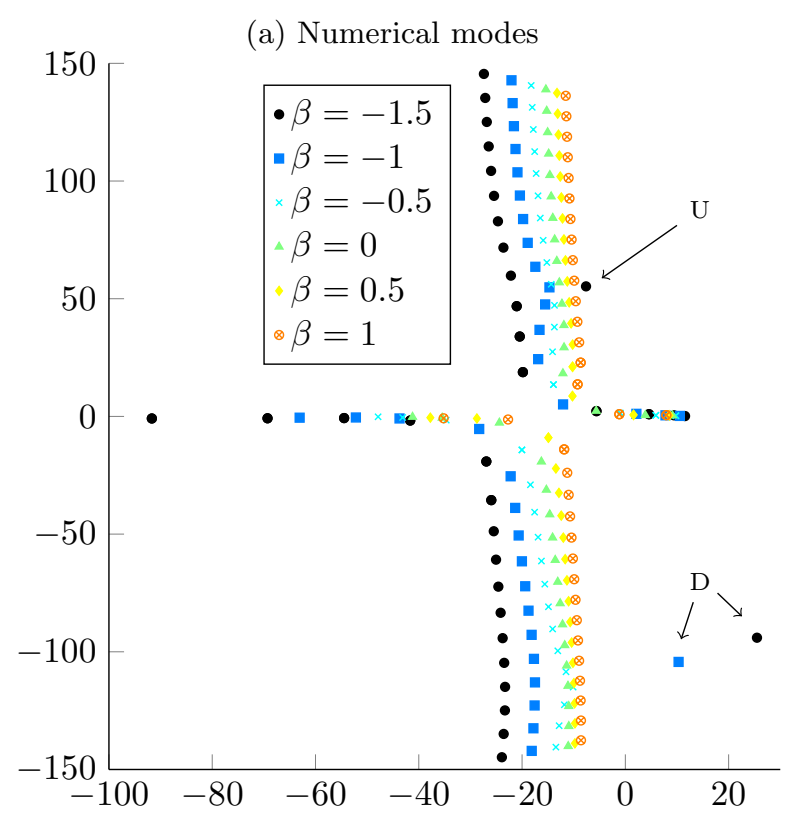

(b) Selection of eigenmodes when $\beta=-1.5$.

\begin{tabular}{|c|c|c|}
\hline & Numerical & Asymptotic \\
\hline \multirow{5}{*}{ 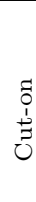 } & $-91.69-0.877 \mathrm{i}$ & $-91.72-0.8769 \mathrm{i}$ \\
\hline & $-69.29-0.7952 \mathrm{i}$ & $-69.32-0.7951 \mathrm{i}$ \\
\hline & $4.582+0.9333 \mathrm{i}$ & $4.597+0.9313 \mathrm{i}$ \\
\hline & $9.415+0.4665 \mathrm{i}$ & $9.411+0.4613 \mathrm{i}$ \\
\hline & $11.56+0.1457 \mathrm{i}$ & $11.54+0.1404 \mathrm{i}$ \\
\hline \multirow{4}{*}{ Ũ } & $-19.84+18.81 \mathrm{i}$ & $-19.82+18.77 \mathrm{i}$ \\
\hline & $-26.92-19.15 \mathrm{i}$ & $-26.89-19.10 \mathrm{i}$ \\
\hline & $-20.45+33.94 i$ & $-20.44+33.91 \mathrm{i}$ \\
\hline & $-25.96-35.57 \mathrm{i}$ & $-25.96-35.53 \mathrm{i}$ \\
\hline |되 & $-7.613+55.25 \mathrm{i}$ & $-7.631+55.24 \mathrm{i}$ \\
\hline 1 & $25.49-94.02 \mathrm{i}$ & $25.49-94.02 \mathrm{i}$ \\
\hline
\end{tabular}

Figure 4: Comparison between numerical and asymptotic eigenmodes as the entropy $s_{0}(r)=-\log \left(r^{\beta}\right)$ varies. The parameters are $\omega=25, \eta=0.6, U_{x}=0.5, U_{\theta}=0, h=0.6$ and lined walls of impedance $Z_{j}=1-2 \mathrm{i}$. The upstream and downstream surface modes are labelled by $U$ and $D$ respectively.

the numerical and asymptotic eigenmodes display all three features, with very small error between the two sets of eigenmodes.

In Figure 4 we see the development of the upstream and downstream surface modes. These surface modes are the analogue of the surface modes given in Rienstra 34 and Brambley and Peake 3 for uniform, non-swirling, homentropic flow. As we decrease the entropy parameter $\beta$, the usually straight branch of cut-off modes pinches and then splits of an eigenmode. As we further decrease the entropy gradient, the

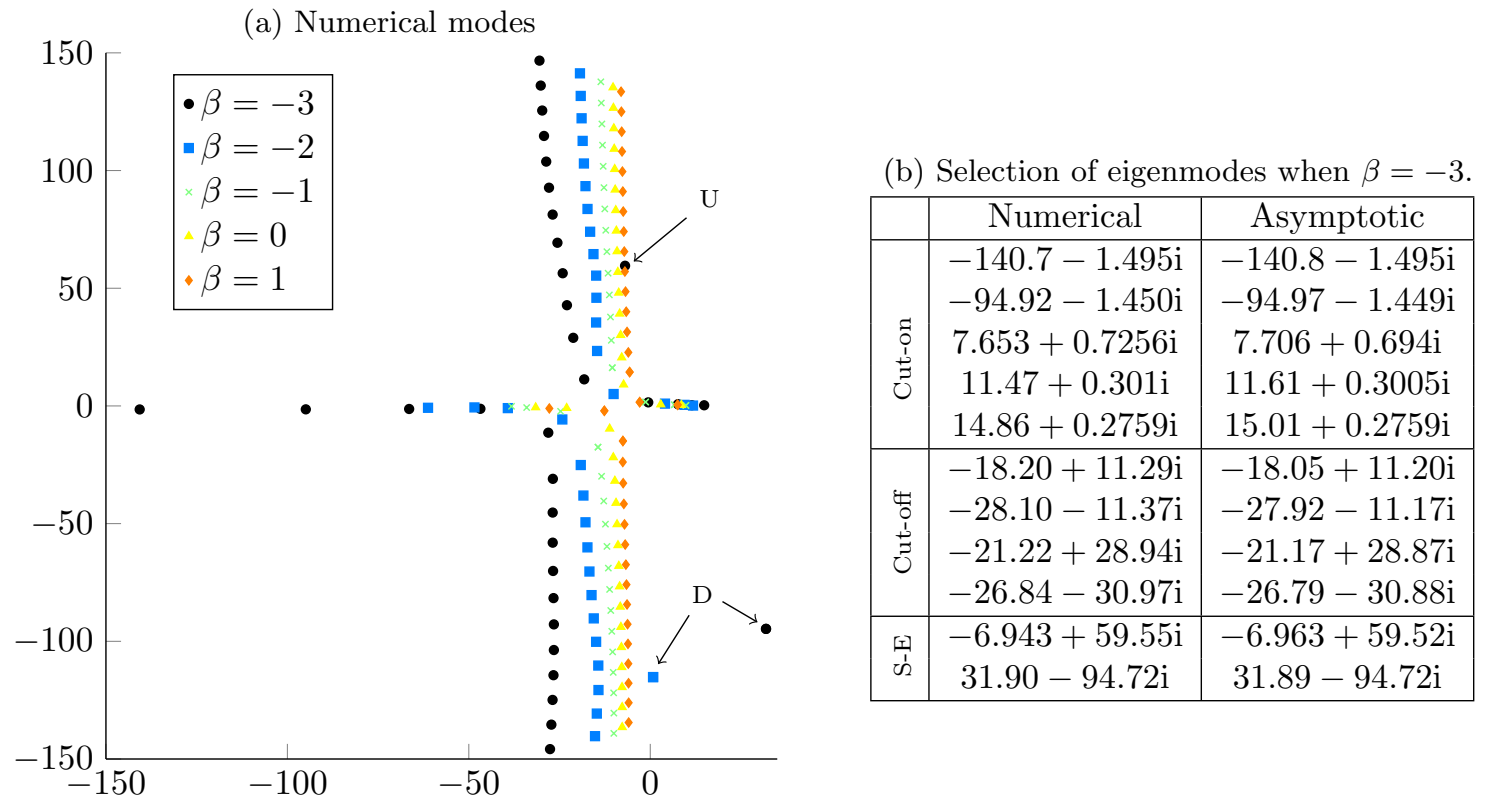

Figure 5: Comparison between numerical and asymptotic eigenmodes as the entropy $s_{0}(r)=-\log \left(r^{\beta}\right)$ varies. The parameters are $\omega=25, \eta=0.48, U_{x}(r)=0.3+0.2 r^{2}, U_{\theta}(r)=0.2 r+0.1 / r, h=0.6$ and lined walls of impedance $Z_{j}=1-2 \mathrm{i}$. The upstream and downstream surface modes are labelled by $U$ and $D$ respectively. 
(a) Numerical modes

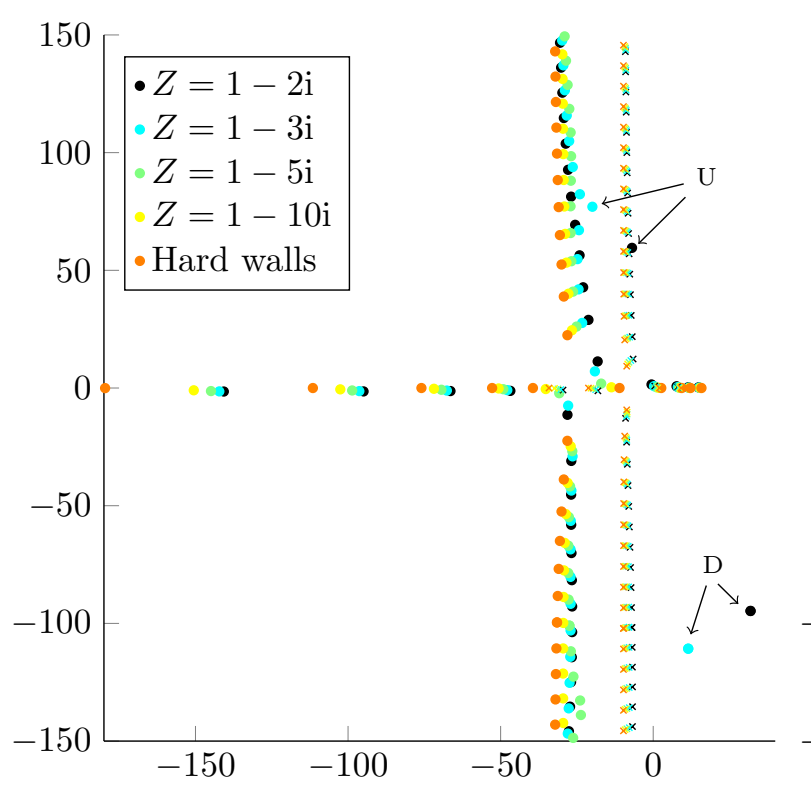

(b) Asymptotic modes

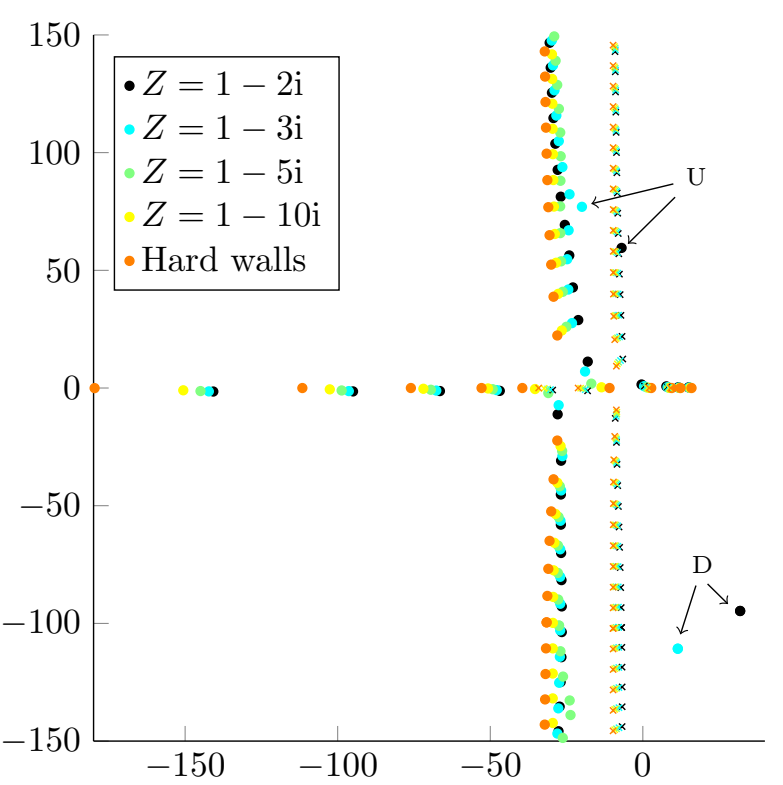

Figure 6: Comparison between numerical and asymptotic eigenmodes, for $\beta=-3$ (circles) and $\beta=0.5$ (crosses). The parameters are $\omega=25, \eta=0.48, U_{x}(r)=0.3+0.2 r^{2}, U_{\theta}(r)=0.2 r+0.1 / r$ and lined walls of different impedances. The upstream and downstream surface modes are labelled by $U$ and $D$ respectively.

branch of cut-off modes straightens again while the surface mode moves right. The downstream surface mode appears for $-1.5<\beta<-1$, while the upstream surface mode appears for $-1<\beta<-0.5$, so at certain values of the entropy gradient there is only one surface mode. The trajectory of the upstream surface mode means that for sufficiently negative entropy gradients, this mode would eventually cross the real line, which would mean the integration contour in Figure 3 would no longer be valid. In Figure 5 we also see these surface modes for polynomial swirling flow, but we have to consider more negative values of $\beta$. We find the upstream mode for $-2<\beta<-1$ and the downstream mode for $-3<\beta<-2$, and again the upstream surface mode would eventually cross the real line. As we have seen, the threshold for the surface mode to develop will be a function of the swirling flow profile, frequency and azimuthal number.

In Figure 6 we see these surface modes disappear as we reduce the lining of the walls by increasing the impedance. This effect is also present when we consider a non-swirling flow such as the flow in Figure 4.

In Figure 4 we see that when $\beta=-1.5$ there are four cut-on downstream modes and four upstream cut-on modes. When $\beta=0$ there are three cut-on downstream modes and three upstream cut-on modes, while when $\beta=1$ there are only two of each. Additionally, the upstream cut-on modes move further and further downstream as we increase the entropy gradient. When $\beta=-1.5$ the furthest upstream cut-on mode is given numerically by $k=-91.69$ (to $2 \mathrm{dp}$ ), while when $\beta=1$ the furthest upstream cut-on mode is given numerically by $k=-35.19$. In contrast, the downstream cut-on modes only move a small amount upstream as we increase the entropy gradient. In Figure 4 the furthest downstream mode is numerically given by $k=11.56$ when $\beta=-1.5$ and given by $k=7.93$ when $\beta=1$. Again, this effect is mainly due to the local Mach number varying rather than a direct result of the varying entropy gradients.

In Figure 5 we see much the same story in terms of cut-on modes, with the number of cut-on modes decreasing as we increase the entropy gradient. When $\beta=-3$ we have four downstream and four upstream cut-on modes, while when $\beta=1$ we have only two downstream and two upstream cut-on modes. In Figures 4 and 5 we see that the branch of cut-off modes moves to the right as we increase the entropy gradient, with the parameters of the flow determining exactly how much the line moves. In Figure 6 we see the effect of entropy with and without lining. Even in a hard-walled duct, we still see fewer cut-on modes and the branch of cut-off modes shifting right as we increase the entropy gradient. 
Table 1: Change in attenuation $(\Delta a)$ for the downstream (numerical) modes as we consider logarithm entropy profiles with different values of $\beta$, and entropy corresponding to constant speed of sound (CSS). We have given the reference attentuation for constant entropy in the first column. Flow parameters as in Figure 6

\begin{tabular}{|l|c|c|c|c|}
\hline $\begin{array}{l}\text { Downstream mode index } \\
(\text { attenutation } a \text { at constant entropy) }\end{array}$ & $\beta=-2$ & $\beta=0$ & $\beta=1$ & CSS \\
\hline $1(-3.06 \mathrm{~dB})$ & 1.702 & 0 & -1.090 & -0.130 \\
$2(-5.26 \mathrm{~dB})$ & 1.326 & 0 & -8.701 & -0.374 \\
$3(-78.72 \mathrm{~dB})$ & 70.203 & 0 & -46.203 & -4.559 \\
$4(-178.66 \mathrm{~dB})$ & 134.707 & 0 & -18.948 & -1.979 \\
\hline
\end{tabular}

In Table 1 we compare the change in attenuation of the downstream (numerical) eigenmodes for different choices of entropy. We use

$$
a=20 \log _{10} \exp \left(\operatorname{Im}\left(k_{\text {ref }}-k\right)\right),
$$

to measure the attenuation (in $\mathrm{dB}$ per unit radius), where $k_{\text {ref }}$ is the eigenmode at constant entropy. We see that for the logarithmic entropy, as we reduce the entropy gradient $\beta$ then we increase the attenuation of all of the modes. Some of the cut-off modes (the third and fourth downstream modes) at constant entropy become cut-on or much close to being cut-on at $\beta=-2$, which massively increases the attenuation. We expect the attenuation to increase as we reduce $\beta$ to be a general effect for all swirling flows for logarithm entropy, and to also apply for the upstream modes. For the entropy corresponding to constant speed of sound, we have a small but noticeable decrease of the attenuation rate for all the downstream modes, which is in the order of a $5 \%$ effect on the attenuation rate of the two cut-on modes.

Finally, we comment on the accuracy of the asymptotic eigenmodes compared with the numerical modes. We find every numerical eigenmode asymptotically, and approximate them extremely accuracy. We are even able to approximate the surface modes with very good accuracy. In Figure 4 , when $\beta=-1.5$, the downstream surface mode is given numerically by $k=-7.61+55.25 \mathrm{i}$ (to $2 \mathrm{dp}$ ) and asymptotically by $k=-7.63+55.24 \mathrm{i}$. The upstream surface mode is given both numerically and asymptotically by $k=$ $25.49-94.02 \mathrm{i}$ to 2 decimal places. In Figure 5, when $\beta=-3$, the downstream surface mode is given numerically by $k=-6.94+59.55 \mathrm{i}$ and asymptotically by $k=-6.96+59.52 \mathrm{i}$. The upstream surface mode is given numerically by $k=31.90-94.72 \mathrm{i}$ and asymptotically by $k=31.89-94.72 \mathrm{i}$. The other asymptotic modes have an accuracy comparable to Mathews and Peake 26.

In Figure 7 we plot the numerical pressure eigenfunctions for the cut-off eigenmodes in Figure 4 when $\beta=-1.5$. The eigenfunctions associated with the surface modes (in red, dashed and dotted) have a considerably different shape to the rest of the eigenfunctions of the cut-off acoustic modes (in orange, solid), since they only oscillate near the inner wall, and decay in the bulk of the flow. It may well be that for other

(a)

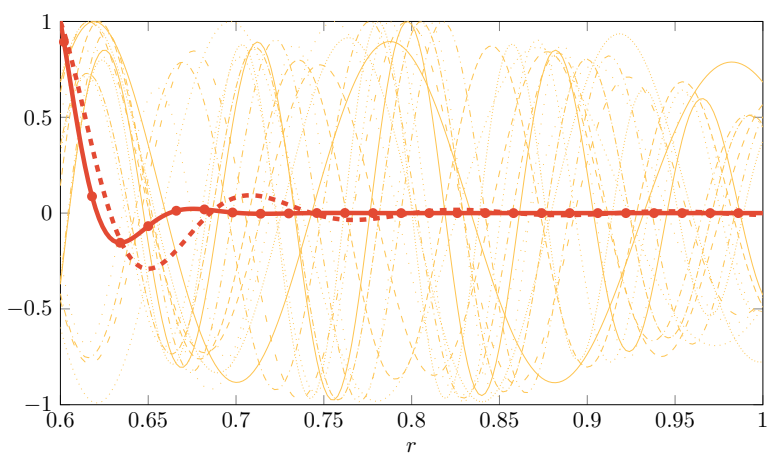

(b)

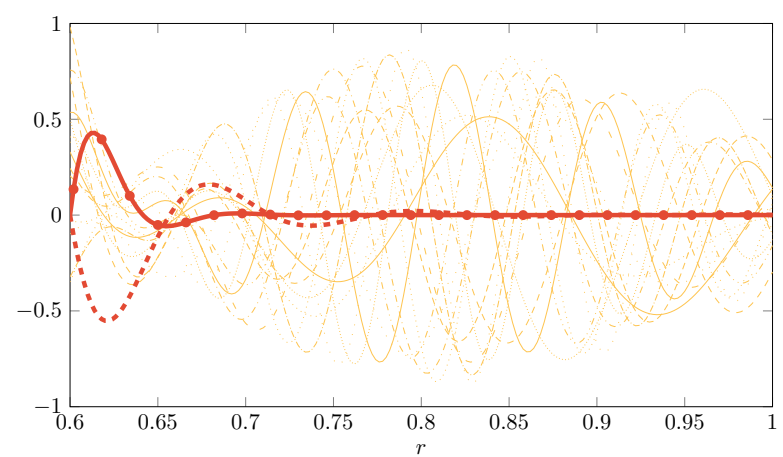

Figure 7: Real (a) and imaginary part (b) of numerical pressure eigenfunction (normalised by $1 / p\left(r_{\max }\right)$ where $r_{\text {max }}$ maximises $\max |p(r)|)$ for different eigenmodes for the parameters in Figure 4 when $\beta=-1.5$. Red, dashed: surface mode $k=-7.61+$ $55.25 \mathrm{i}$, red, solid with circles: surface mode $k=25.49-94.02 \mathrm{i}$, orange, solid: other cut-off modes. 
flow regimes we get the oscillation of surface waves at the outer wall rather than the inner wall.

\section{Effect of entropy on hydrodynamic modes}

We now consider the effect of a swirling base flow with varying entropy gradient on the hydrodynamic modes at the ends of the critical layer. Although we believe the Green's function contribution from these mode is generally very small [32], for completeness we investigate them here. Using Eq. (56) we find the Fourier transform of pressure, denoted $P$, satisfies the equation

$$
\frac{\mathrm{d}^{2} P}{\mathrm{~d} r^{2}}+\mathcal{B}(r) \frac{\mathrm{d} P}{\mathrm{~d} r}+\mathcal{C}(r) P=0
$$

where $\mathcal{B}$ is given by

$$
\mathcal{B}:=\left[\left(\frac{1}{r}-\frac{\rho_{0}^{\prime}}{\rho_{0}}\right)-\frac{D^{\prime}}{D}\right]
$$

and

$$
\mathcal{C}:=\frac{1}{\Omega^{2}}\left(D\left(\frac{\Omega^{2}}{c_{0}^{2}}-k^{2}-\frac{n^{2}}{r^{2}}\right)-\Upsilon\left[\Upsilon+\Omega\left(\frac{1}{r}-\frac{\rho_{0}^{\prime}}{\rho_{0}}\right)\right]-\Upsilon \frac{[\Omega D]^{\prime}}{D}-\Omega \Upsilon^{\prime}\right)
$$

In Eq. 82 and Eq. $83 D(r)=\Omega^{2}(r)-\mathcal{U}_{\theta}(r)$, with $\mathcal{U}_{\theta}$ defined in Eq. 39 , while the functions $\Omega$ and $\Upsilon$ are defined in Eq. (57). Our definition of $D$ matches Heaton and Peake [17, while $\Omega$ is denoted by $-\Lambda$ in Heaton and Peake [17] and $\Upsilon$ has no equivalent term. Given a critical radius $r_{c}$, we can define $k_{c}(r)$ such that $\Omega\left(k_{c}\left(r_{c}\right), r_{c}\right)=0$, with

$$
k_{c}(r)=\frac{1}{U_{x}(r)}\left[\omega-\frac{n U_{\theta}(r)}{r}\right] .
$$

\subsection{Asymptotic method}

We use the Frobenius method [1] to solve the differential equation asymptotically near a critical point and to do this we first write $\mathcal{B}(r)$ and $\mathcal{C}(r)$ as Laurent series about $r_{c}$. We find that the leading terms in the series occur at $1 / r$ and $1 / r^{2}$ respectively, with coefficients

$$
\mathcal{B}_{-1}=0 \text { and } A(r):=\mathcal{C}_{-2}=\frac{2 U_{\theta} k_{c}\left[k_{c}\left(U_{\theta} r\right)^{\prime}-n U_{x}^{\prime}\right]+\mathcal{E}}{r^{2}\left[n\left(U_{\theta} / r\right)^{\prime}+k_{c} U_{x}^{\prime}\right]^{2}}
$$

where

$$
\mathcal{E}(r)=r^{2} \frac{U_{\theta}^{2}(r)}{r}\left(\frac{\rho_{0}^{\prime}(r)}{\rho_{0}(r)}-\frac{U_{\theta}^{2}(r)}{r c_{0}^{2}(r)}\right)\left(k_{c}^{2}+\frac{n^{2}}{r^{2}}\right) .
$$

Where we have a homentropic base flow, $\mathcal{E}(r)$ is zero since the first bracketed term vanishes, and hence $A(r)$ matches the definition in Heaton and Peake [17. Although Lalas [20] considered a flow with entropy, he did not consider the Frobenius expansion so this is a new result. From Lalas [20, Heaton and Peake [17] we see that the entropy term $\mathcal{E}(r)$ is related to the Brunt-Väisälä frequency.

The indicial equation in the Frobenius method becomes $\sigma^{2}-\sigma+A\left(r_{c}\right)=0$, where $\sigma$ is the indicial exponent, and it has solutions

$$
\sigma_{ \pm}=\frac{1}{2} \pm \mathrm{i} \lambda\left(r_{c}\right) \text { where } \lambda\left(r_{c}\right)=\sqrt{A\left(r_{c}\right)-\frac{1}{4}}
$$

When $A\left(r_{c}\right)>1 / 4$ we find the Frobenius (inner) solution is given, to leading order, by

$$
P_{I}(r)=c_{P}\left(r-r_{c}\right)^{1 / 2}\left[\sin \left(\lambda\left(r_{c}\right) \log \left(r-r_{c}\right)+\mu\right)\right]
$$


with $\mu$ a constant. Since the unsteady potential $\varphi$ is related to the pressure by

$$
p=-\rho_{0} \frac{D_{0} \varphi}{D t},
$$

we then find that the Fourier transform of the unsteady potential, $\phi$, satisfies

$$
P(r)=\mathrm{i} \rho_{0}(r) \Omega(r) \phi(r) \approx \mathrm{i} \rho_{0}\left(r_{c}\right) \Omega^{\prime}\left(r_{c}\right)\left(r-r_{c}\right) \phi(r),
$$

and hence to leading order the inner, Frobenius solution for $\phi$ is

$$
\phi_{I}(r)=c_{\phi}\left(r-r_{c}\right)^{-1 / 2}\left[\sin \left(\lambda\left(r_{c}\right) \log \left(r-r_{c}\right)+\mu\right)\right],
$$

as in Heaton and Peake 17 .

We now apply exactly the same analysis as in Heaton and Peake [17 to determine the hydrodynamic modes asymptotically. We match the inner solution $P_{I}$ or $\phi_{I}$ to an outer solution (in some cases via an intermediate solution), and then apply the boundary conditions at the duct walls. This gives allowed values of $r_{c}$, which will give eigenmodes $k_{c}\left(r_{c}\right)$. We are only interested in values of $r_{c}$ which give eigenmodes close to the two ends of the critical layer. The value of $r_{c}$ (and hence $k_{c}$ ) will be determined by $\lambda$, which depends on the entropy through the term $\mathcal{E}(r)$.

\subsection{Summary of asymptotic results}

Case 1: $k_{c}(r)$ is monotonic and $A(r)>1 / 4$.

In case $1 r_{c}$ is close to $r=h$ or $r=1$ but outside of the duct. We find the hydrodynamic modes accumulate at an exponential rate of

$$
\exp \left(-\frac{m \pi}{\lambda(1)}\right) \text { or } \exp \left(-\frac{m \pi}{\lambda(h)}\right)
$$

depending on at which end of the critical layer they accumulate. Thus, the modes are asymptotically given by

$$
k_{m}^{ \pm} \sim k_{c}(1) \pm c_{1} \exp \left(-\frac{m \pi}{\lambda(1)}\right) \text { and } k_{m}^{\mp} \sim k_{c}(h) \mp c_{h} \exp \left(-\frac{m \pi}{\lambda(h)}\right), m \in \mathbb{N} .
$$

The \pm comes from whether $k_{c}$ is increasing or decreasing, with $k_{m}^{+}$to the right of the critical layer and $k_{m}^{-}$ to the left of the critical layer. We only get accumulation at the duct walls when $A(r)>1 / 4$ ( $\lambda$ is real), and it is possible that the modes accumulate at one end of the duct and not at the other.

If we follow the method in Heaton and Peake [17, then we could deduce the constants $c_{1}$ and $c_{h}$. However, it was shown in Mathews 25] that the method is not fully correct, and fails because Heaton and Peake assume the composite solution of the inner and the outer solution is sinusoidal, when it is Airy-like. Furthermore, it was shown 25] that the constants $c_{h}$ and $c_{1}$ are global properties of the flow, and depend on the flow in a region near the duct wall, rather that at a single point. Thus, these constants have to be determined numerically.

Case 2: $k_{c}(r)$ has a single critical point $r=r^{\star}$, solving $k_{c}^{\prime}\left(r^{\star}\right)=0$. Additionally, $A\left(r^{\star}\right)>0$.

In case $2 k_{c}(r)$ is not monotonic and we look for critical points $r_{c}$ near $r=r^{\star}$ and one of $r=h$ and $r=1$. The modes accumulate at an algebraic rate in the real plane near $k_{c}\left(r^{\star}\right)$, with

$$
\left.\left|k_{m}-k_{c}\left(r^{\star}\right)\right| \sim \frac{1}{m^{2}}\left(\frac{2}{-k_{c}^{\prime \prime}\left(r^{\star}\right)}\right) \frac{2 U_{\theta} k_{c}\left[k_{c}\left(U_{\theta} r\right)^{\prime}-n U_{x}^{\prime}\right]+\mathcal{E}(r)}{r^{2} U_{x}^{2}}\right|_{r=r^{\star}} .
$$

At the other end of the critical layer, the modes accumulate exponentially if $A(r)>1 / 4$ (and otherwise not at all), with accumulation rate given by Eq. 92.

Case 3: $k_{c}(r)$ has a single critical point $r=r^{\star}$, solving $k_{c}^{\prime}\left(r^{\star}\right)=0$. Additionally, $A\left(r^{\star}\right)<0$. 
In case $3 k_{c}(r)$ is not monotonic and we again look for critical points $r_{c}$ near $r=r^{\star}$ and one of $r=h$ and $r=1$. The modes accumulate at an algebraic rate in the complex plane near $k_{c}\left(r^{\star}\right)$, with

$$
k_{m}-\left.k_{c}\left(r^{\star}\right) \sim \frac{1}{m^{2}}\left(\frac{2}{k_{c}^{\prime \prime}\left(r^{\star}\right)}\right) \frac{2 U_{\theta} k_{c}\left[k_{c}\left(U_{\theta} r\right)^{\prime}-n U_{x}^{\prime}\right]+\mathcal{E}(r)}{r^{2} U_{x}^{2}}\right|_{r=r^{\star}}+c_{I} \frac{\mathrm{i}}{m^{3}},
$$

when the right edge of the critical layer is given by $k_{c}\left(r^{\star}\right)$, and $c_{I}$ is a constant of proportionally that is not determined in Heaton and Peake [17]. A similar result holds when the left edge of the critical layer is given by $k_{c}\left(r^{\star}\right)$. At the other end of the critical layer the modes accumulate exponentially if $A(r)>1 / 4($ and otherwise not at all), with accumulation rate given by Eq. (92).

\subsection{Applicability of asymptotic results}

Although we can determine the constant of proportionality when the modes accumulate algebraically on the real line (case 2), knowing it is not very enlightening. It takes of the order of 100 modes [17, Section 3.4] before the modes accumulate algebraically and the relation

$$
k_{m}^{+}=k_{c}\left(r^{\star}\right)+\frac{c_{A}}{m^{2}}, \quad m \in \mathbb{N},
$$

where $c_{A}$ is given in Eq. (94), is accurate. In contrast, for modes accumulating exponentially, relations of the form in Eq. (93) where $c_{1}$ and $c_{h}$ are to be determined, are accurate after only about ten modes. If we calculate the first ten or so hydrodynamic modes numerically, then we can determine $c_{1}$ and $c_{h}$ and hence the rest of the modes asymptotically.

Thus, we will always need to calculate some of the hydrodynamic modes numerically, in the first case because we don't know the constant of proportionality and in the second and third cases because the first asymptotic modes are not accurate. Further, we would need to calculate significantly more of them numerically when they accumulate algebraically than when then accumulate exponentially to get accurate results.

\subsection{Numerical results}

We now consider the effect of entropy on the hydrodynamic modes. We calculate these modes numerically, using either Chebfun [9] or using the program from Heaton and Peake [17]. We consider a base flow entropy of the form $s_{0}(r)=-\log \left(r^{\beta}\right)$, and mainly consider the three cases $\beta=-0.3, \beta=0$ and $\beta=0.3$. For this choice of entropy we calculate that

$$
\mathcal{E}(r)=\frac{\beta}{c_{p}} U_{\theta}^{2}(r)\left(k_{c}^{2}(r)+\frac{n^{2}}{r^{2}}\right)
$$

In Figure 8 we plot the hydrodynamic modes for a base flow with uniform axial flow and swirling flow consisting of solid body and rotation and a free-vortex, and we see that the modes move as we vary entropy.

We see that for this choice of parameters, larger values of $\beta$ (and hence larger entropy gradients) shift all the hydrodynamic modes right at the right end of the critical layer. We find at the other end of the critical layer that as we increase the entropy gradient the modes shift left. Thus, at both ends the hydrodynamic modes shift away from the critical layer as we increase the entropy gradient. In Figure 8 we also calculate the values of $\lambda(1)=\sqrt{A(1)-1 / 4}$, where $A(r)$ is given in Eq. 85 . Since we are in the first case, the modes accumulate at an exponential rate, and hence at the right edge of the critical layer they are asymptotically given by

$$
k_{m}^{+} \sim k_{c}(1)+c_{1} \exp \left(-\frac{m \pi}{\lambda(1)}\right), \quad m \in \mathbb{N},
$$

where $c_{1}$ is the constant in Figure 8. We calculate this constant numerically. Thus, in Figure 8 the hydrodynamic modes shift right as we increase the entropy gradient because the constant of proportionality $c_{1}$ increases, while the exponential accumulation rate $\lambda(1)$ also increases as the entropy gradient increases. 


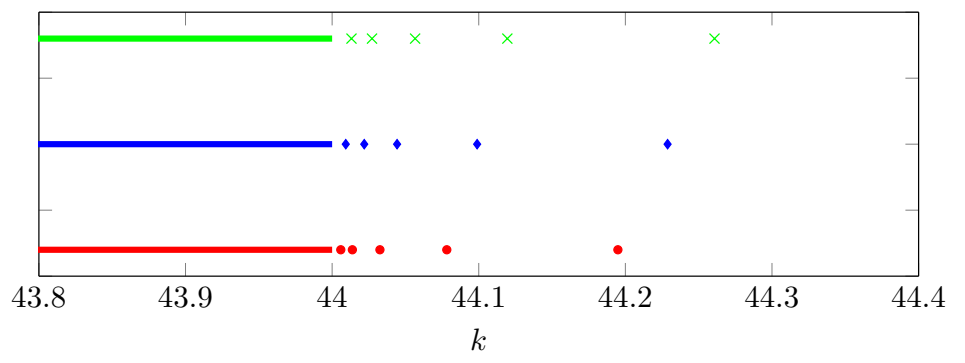

\begin{tabular}{|c|c|c|c|}
\hline & $\beta=-0.3$ & $\beta=0$ & $\beta=0.3$ \\
\hline & 44.0059 & 44.0093 & 44.0132 \\
चै & 44.0138 & 44.0219 & 44.0272 \\
$\frac{\vec{\sigma}}{0}$ & 44.0326 & 44.0443 & 44.0566 \\
$\vec{z}$ & 44.0782 & 44.0989 & 44.1195 \\
& 44.1949 & 44.2288 & 44.2608 \\
\hline$\lambda(1)$ & 3.7539 & 4.1181 & 4.4526 \\
$c_{1}$ & 0.3873 & 0.4217 & 0.4503 \\
\hline
\end{tabular}

Figure 8: Right-hand side of critical layer (solid line) showing first five hydrodynamic modes (marks). The parameters are $U_{x}=0.5, U_{\theta}(r)=0.1 r+0.1 / r, \omega=25, n=15$ and $h=0.6$. Green crosses: $\beta=0.3$, blue diamonds: $\beta=0$ and red circles: $\beta=-0.3$. The critical layer is given by $\mathcal{K}^{C L}=[38.66,44]$.

From the definition of $\lambda(r)$ and $\mathcal{E}(r)$ it is clear why the latter happens, but because we cannot determine the constant of proportionality $c_{1}$ asymptotically it is not clear of the exact effect $\beta$ has on the constant of proportionality.

In Figure 9 we show the other two cases for accumulation at the edge of the critical layer. We take the flows given in Heaton and Peake [17] and consider them with different base flow entropies by varying $\beta$. In Figure 9 a we see the modes accumulating algebraically on the real line. As we increase the entropy gradient the accumulation rate in Eq. (94) varies due to the $\beta$ dependence which causes the hydrodynamic modes to all shift to the right.

In Figure 9b we see the first few hydrodynamic modes accumulating in the complex plane. As we increase the entropy gradient, the modes move to the left at the left edge of the critical layer, as in the previous two cases. We also see that as we increase the entropy parameter from $\beta=0.3$ (green crosses) to $\beta=0.6$ (orange diamonds) we change accumulation regime and go from clustering in the complex plane to clustering on the real line, which follows from Section 5.2 and the definition of $A(r)$ in terms of the entropy. Thus, increasing the entropy gradient (by increasing $\beta$ ) is stabilising the flow since we have changed accumulation regime [17.

For the choice of logarithm entropy, we see that for any swirling flow regime and frequency, we can always stabilise flows that were accumulating algebraically in the complex plane by making $\beta$ large enough, while we can always destabilise flows that accumulating algebraically in the real plane by making $\beta$ small enough. The threshold value of $\beta$ where it changes stability will depend on the swirling flow regime and frequency. For other choices of entropy, the function $\mathcal{E}(r)$ might have a more complicated dependence on $r$ (as opposed to always being positive or negative, depending on the sign of $\beta$ ), which makes it less clear about the effect of entropy on stability.

(a)

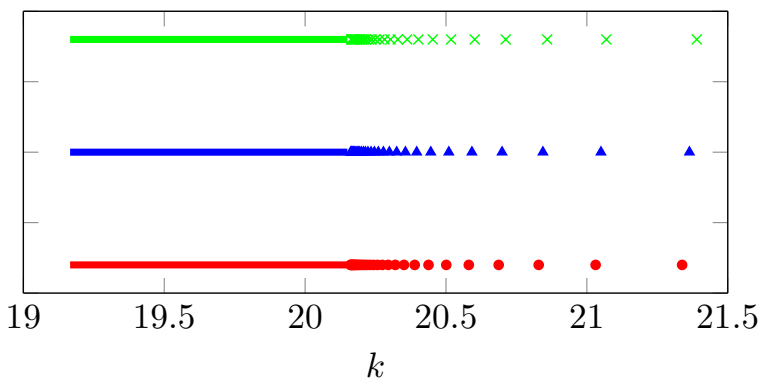

(b)

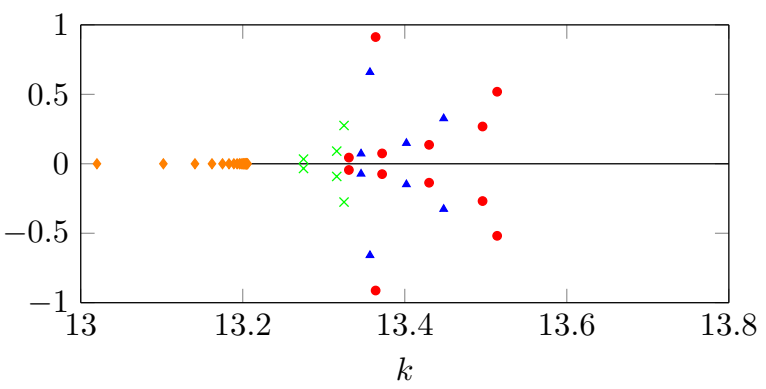

Figure 9: Plot of the hydrodynamic modes as we vary the entropy gradient for different flow parameters. (a) $U_{x}(r)=0.5+0.1 r^{4}$, $U_{\theta}(r)=0.5 r^{4}, h=0.5, n=-3, \omega=10$. (b) $U_{x}(r)=0.7-0.5 r^{2}, U_{\theta}(r)=0.1 r+0.25 / r, h=0.5, n=-5, \omega=3$. We only consider the first few modes in Figure $9 \mathrm{~b}$ Orange diamonds: $\beta=0.6$ (only in Figure 9b), green crosses : $\beta=0.3$, blue triangles: $\beta=0$ and red circles: $\beta=-0.3$. 


\section{Effect of entropy on Green's function}

In Section 4 we saw that the logarithm entropy had three main effects on the eigenmodes; the appearance of surface modes, a shifting of the branch of cut-off modes to the right and a reduction in the number of cut-on modes. Since the main contribution to the acoustic Green's function comes from the cut-on modes, it is the last feature that causes the Green's function to change significantly as we vary entropy.

In Figure 10 we plot the Green's function contributions $\widehat{p}_{n}^{m}$ from the three furthest downstream cut-on eigenmodes as the entropy varies. We also plot the total acoustic Green's function $\widehat{p}_{n}^{A}$. The base flow entropy is given by $s_{0}(r)=-\log \left(r^{\beta}\right)$, with $\beta=-0.3$ (left), $\beta=0$ (middle) and $\beta=0.3$ (right). As we vary the value of $\beta$, the density, speed of sound and pressure of the base flow all vary since the base flow must satisfy the Euler equations. This then causes functions such as $q_{n}(r, \kappa)$ and $\Phi(r, \kappa)$ to vary with the entropy gradient.

We only need to consider the three most cut-on modes in Figure 10 since subsequent upstream modes for all three values of $\beta$ are cut-off, with the Green's function contributions $\widehat{p}_{n}^{m}$ from these cut-off modes orders of magnitude smaller than the contributions from the dominant cut-on modes.

In Figure 10 we see the Green's function contribution $\widehat{p}_{n}^{m}$ from the furthest downstream eigenmode (top row) is very similar for all values of $\beta$. When we consider the next eigenmode (second row) we see that the

(a)

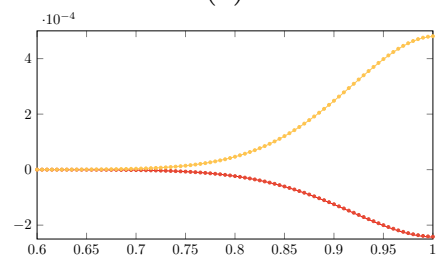

(d)

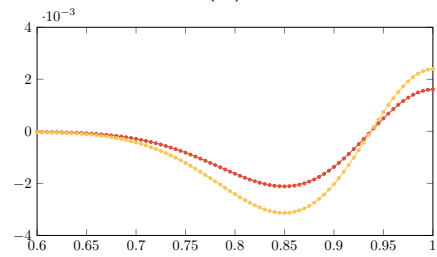

(g)

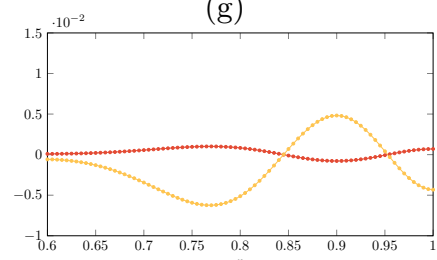

(j)

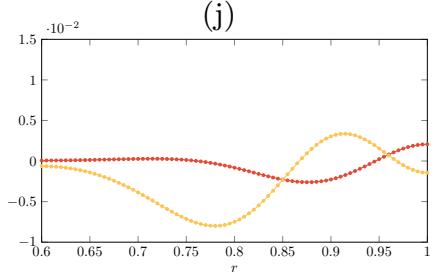

(b)

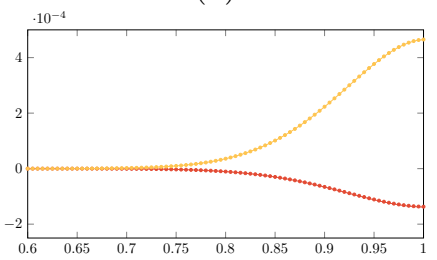

(e)

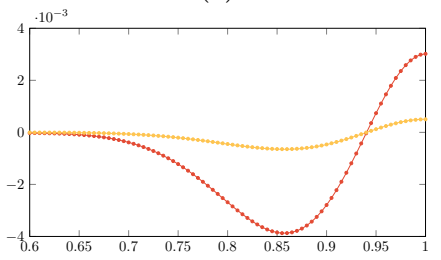

(h)

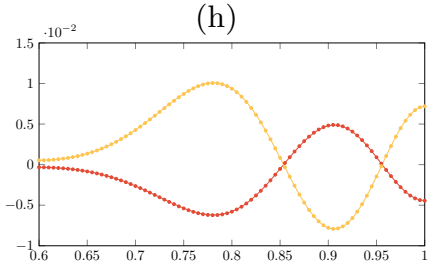

(k)

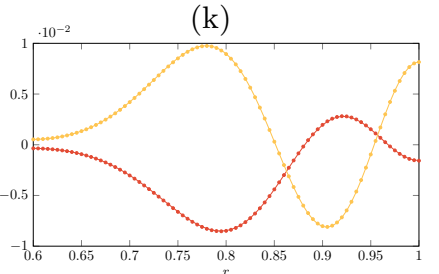

(c)

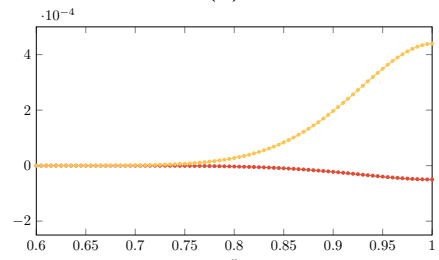

(f)

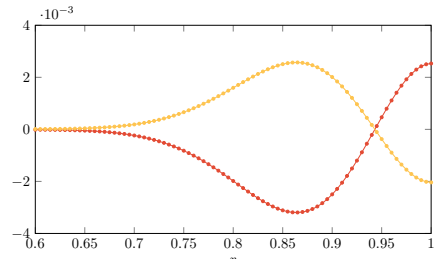

(i)

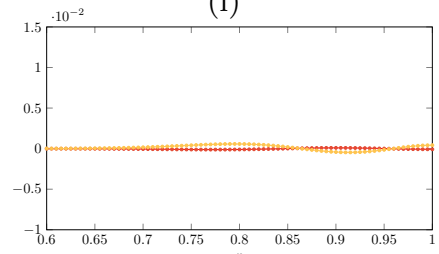

(l)

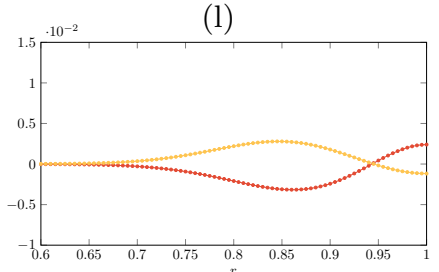

Figure 10: Green's function $\widehat{p}_{n}^{m}$ from the three most cut-on upstream acoustic modes and total acoustic Green's function $\widehat{p}_{n}^{A}$ (top to bottom) as the entropy gradient varies (left to right) for a source at $x-x_{0}=0.5$ and $r_{0}=0.8$. (a) asymptotic mode $k=13.503$, numerical mode $k=13.497$ when $\beta=-0.3$ (d) $k=5.101(5.096),(\mathrm{g}) k=-5.962(-5.962)(\mathrm{j})$ total Green's function. (b) asymptotic mode $k=13.144$, numerical mode $k=13.139$ when $\beta=0$ (e) $k=3.473(3.471)$, (h) $k=-11.465(-11.457)(\mathrm{k})$ total Green's function. (c) asymptotic mode $k=12.796$, numerical mode $k=12.792)$ when $\beta=0.3$ (f) $k=1.786(1.786)$, (i) $k=-21.551+7.187 \mathrm{i}(-21.555+7.171 \mathrm{i})$ (l) total Green's function.

The parameters of the flow are $U_{x}(r)=0.3+0.2 r^{2}, U_{\theta}(r)=0.1 r+0.1 / r, n=32, \omega=50, h=0.6$ and hard walls. Solid lines are the asymptotic Green's function; circles are the numerical Green's function. Red is for the real part, orange is for the imaginary part. 
(a)

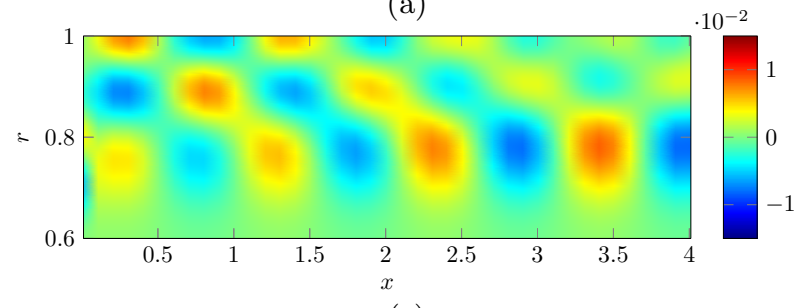

(c)

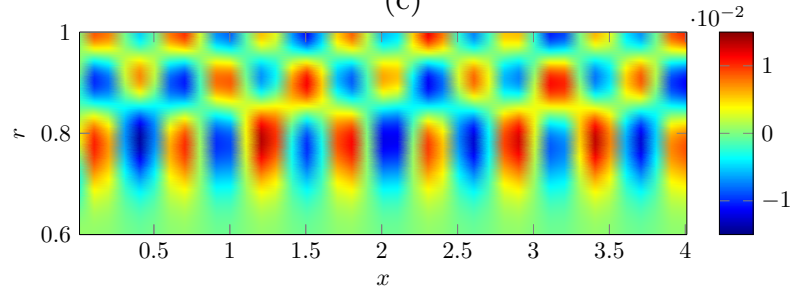

(e)

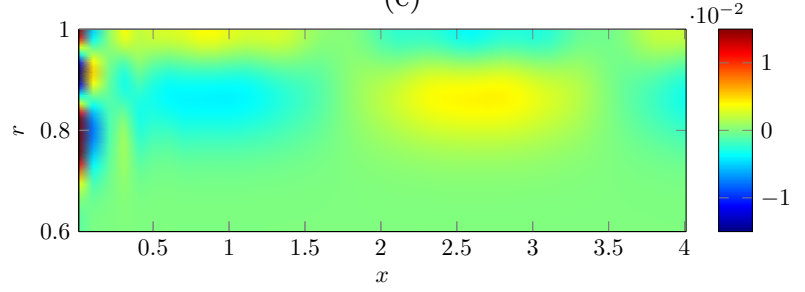

(b)

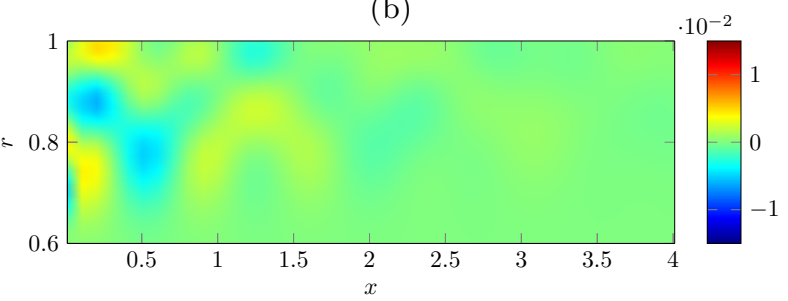

(d)

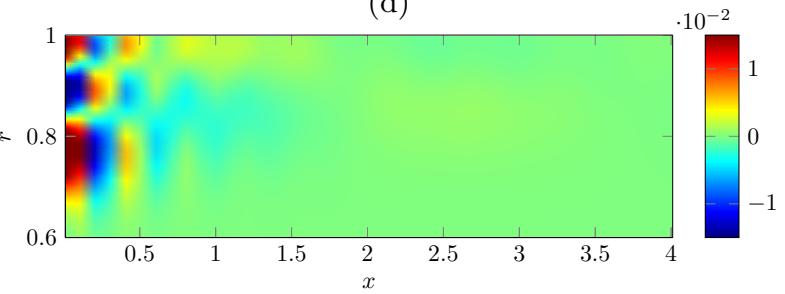

(f)

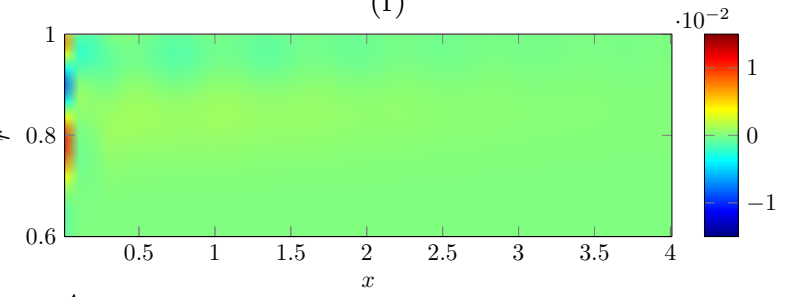

Figure 11: Colour plot of real part of asymptotic Green's function $\widehat{p}_{n}^{A}$ with a source at $\left(x_{0}, r_{0}\right)=(0,0.8)$ as $x$ and $r$ vary. (a) $\beta=-0.3$, hard walls, (c) $\beta=0$, hard walls, (e) $\beta=0.3$, hard walls. (b) $\beta=-0.3, Z_{j}=1-2 \mathrm{i},(\mathrm{d}) \beta=0, Z_{j}=1-2 \mathrm{i}$, (f) $\beta=0.3, Z_{j}=1-2 \mathrm{i}$. The other flow parameters are the same as Figure 10 .

shape of the Green's function contribution changes significantly as we vary the entropy gradient. This is because the second downstream eigenmode moves by a significant amount as we vary the entropy gradient.

When we consider the Green's function contribution $\widehat{p}_{n}^{m}$ from the third furthest downstream cut-on eigenmode (third row), we see significantly different shapes and amplitudes for the Green's function. When $\beta=0.3$ this eigenmode is cut-off, while when $\beta=0$ or $\beta=-0.3$ it is cut-on. Finally, in the fourth row we see the total acoustic contribution, $\widehat{p}_{n}^{A}$, obtained by summing the contribution from all the downstream modes, with vastly different Green's functions as the entropy gradient varies. When $\beta=0$ and $\beta=-0.3$ the third furthest downstream eigenmode contributes most to the total Green's function, since it is $\mathcal{O}\left(10^{-2}\right)$. However, when $\beta=0.3$ the second furthest downstream mode is the dominant eigenmode. Thus, varying entropy not only changes the Green's function at each mode, but changes the dominant eigenmode(s).

In Figure 10 we see that for all values of entropy gradient our asymptotic Green's function is very accurate compared to the numerical Green's function. The absolute error in the total acoustic Green's function $\widehat{p}_{n}^{A}$ in Figures $10 \mathrm{j}$ and 101 is $\mathcal{O}\left(10^{-6}\right)$, and $\mathcal{O}\left(10^{-5}\right)$ in Figure $10 \mathrm{k}$, with the relative errors very similar in all three figures.

For the logarithm entropy profile, we expect that as we increase $\beta$ then we will reduce the magnitude of the Green's function universally in any flow regime, since cut-on modes become cut-off. For general entropy profiles this may not be the case, as the relationship between entropy and number of cut-on modes is less clear.

In Figure 11 we plot the real part of the total asymptotic Green's function $\widehat{p}_{n}^{A}$ for a source at $\left(x_{0}, r_{0}\right)=$ $(0,0.8)$ as $x$ and $r$ vary. We see the effect of both entropy and lining on the Green's function. In both a lined duct and a hard-walled duct, we see that when $\beta=0.3$ the Green's function is a lot smaller in magnitude than when $\beta=0$ or $\beta=-0.3$, since the dominant eigenmode becomes cut-off. It is clear that the Green's function is very different for the three values of entropy gradient. As the entropy gradient varies, we have three completely different colour plots, and the period in the axial direction varies significantly with entropy for both hard and lined duct walls. 
The lining causes the Green's function to decay in the axial direction for all values of entropy gradient. Additionally, in a lined duct the Green's function has a significantly smaller magnitude that in a hard-walled duct away from the source. Close to the source, the Green's function can have a similar or larger magnitude. In Figures 11c and 11d we see that the Green's function in a lined duct had a larger magnitude close to the source, with the colour map saturated in Figure $11 \mathrm{~d}$.

It is clear from our results that a small difference in entropy can modify the Green's function substantially. Thus, we need to model the base flow entropy accurately so we calculate the correct Green's function.

\section{Conclusion}

We began by deriving a new acoustic analogy, given in Eq. 477), by rearranging the Euler equations for a swirling, isentropic base flow, extending the result from Posson and Peake 32 . When we considered the acoustic analogy with constant base flow entropy, we got the same operator $\mathcal{F}$ acting on the pressure perturbation as in Posson and Peake [32. The source terms are also equivalent for a homentropic base flow, although in slightly different forms.

Our new acoustic analogy allowed us to see the effect of entropy on the eigenmodes and Green's function, both numerically and analytically. Our analytic results were extremely accurate, and of a similar accuracy to the case of homentropic flow in Mathews and Peake [26. We saw that in the high-frequency limit varying the base flow entropy only affects the base flow pressure, density and speed of sound; the dispersion relations for the eigenmodes and the Green's function do not otherwise change. If we made the base flow entropy gradient sufficiently negative we could find both an upstream and a downstream surface mode (Figures 4 and 5), which were created by a mode splitting off from the main line of cut-off modes. These surface modes were present regardless of the swirl of the flow, but were only present when there was lining in the duct. We were able to accurately approximate the eigenmodes analytically in swirling flow with variable entropy (Figures 4 to 6), including when surface modes were present. We found that the analytic Green's function for a base flow with varying entropy was very accurate compared to the numerical Green's function, as seen in Figure 10. In Figure 11 we saw that the base flow entropy affects the Green's function significantly, for both a hard-walled and a lined duct.

We also considered the calculation of the hydrodynamic modes accumulating at the end of the critical layer analytically. We extended the analytic results from Heaton and Peake [17] to a base flow with non constant entropy in Eq. (92) to Eq. (95) and then compared to numerical results. For the parameters we tested, increasing the entropy resulted in the hydrodynamic modes moving further away from the critical layer (Figures 8 and 9). We also came to the conclusion that we would always have to calculate some of the hydrodynamic modes numerically, in the first case because we don't know the constant of proportionality and in the second and third cases because the first analytical modes are not accurate.

\section{Acknowledgements}

Some of the initial ideas of this paper were presented as part of the conference paper Mathews et al. 27, AIAA paper 2016-2922 at the 22nd AIAA/CEAS Aeroacoustics Conference, 30 May - 1 June 2016, Lyon, France.

This work was supported by the UK Engineering and Physical Sciences Research Council (EPSRC) grant EP/H023348/1 for the University of Cambridge Centre for Doctoral Training, the Cambridge Centre for Analysis.

\section{Appendix A.}

We now define the functions $\mathscr{A}^{j}(r, \kappa), \mathscr{B}^{j}(r, \kappa), \mathbb{A}^{j}(r, \kappa)$ and $\mathbb{B}^{j}(r, \kappa)$ used in Section 3.2 . These were previously derived in Mathews [25], Mathews and Peake [26]. We firstly consider the case when $q_{n}(r, \kappa)$ has 
either no zeros or a single zero $r_{c} \in \mathcal{R}$ with $q_{n}\left(r_{c}, \kappa\right)=0$, where the region $\mathcal{R}$ is defined as

$$
\mathscr{R}=\left\{r \in \mathbb{C}|| r-s \mid<\omega^{-2 / 3} \text { for } s \in[h, 1]\right\} .
$$

We first define

$$
\tau_{r}(\kappa)=\left(\frac{3 \omega \Psi_{n}(r, \kappa)}{2}\right)^{2 / 3}, \quad \psi_{r}(\kappa)=\int_{h}^{r} \sqrt{q_{n}(s, \kappa)} \mathrm{d} s \text { and } \Psi_{r}(\kappa)=\int_{r_{c}}^{r} \sqrt{q_{n}(s, \kappa)} \mathrm{d} s .
$$

Additionally we define $q_{r}(\kappa)=q_{n}(r, \kappa)$, and let $\mathbb{1}$ denote the indicator function. We then have

$$
\begin{aligned}
& \mathscr{A}(r, \kappa)=\frac{1}{1+\mathrm{i}}\left(q_{r}(\kappa)\right)^{-1 / 4} e^{\mathrm{i} \omega \psi_{r}(\kappa)} \mathbb{1}_{\mathscr{K} C}+\sqrt{\pi}\left[\left(\frac{\tau_{r}(\kappa)}{q_{r}(\kappa)}\right)^{1 / 4} \operatorname{Ai}\left(-\tau_{r}(\kappa)\right)\right] \mathbb{1}_{\mathscr{K}}, \\
& \mathscr{B}(r, \kappa)=\frac{1}{1+\mathrm{i}}\left(q_{r}(\kappa)\right)^{-1 / 4} e^{-\mathrm{i} \omega \psi_{r}(\kappa)} \mathbb{1}_{\mathscr{K}^{C}}+\sqrt{\pi}\left[\left(\frac{\tau_{r}(\kappa)}{q_{r}(\kappa)}\right)^{1 / 4} \operatorname{Bi}\left(-\tau_{r}(\kappa)\right)\right] \mathbb{1}_{\mathscr{K}}, \\
& \mathbb{A}(r, \kappa)=\frac{1}{1+\mathrm{i}}\left(-\frac{\frac{\partial}{\partial r} q_{r}(\kappa)}{4 q_{r}(\kappa)}+\mathrm{i} \omega \sqrt{q_{r}(\kappa)}\right)\left(q_{r}(\kappa)\right)^{-1 / 4} e^{\mathrm{i} \omega \psi_{r}(\kappa)} \mathbb{1}_{\mathscr{K}^{C}}+\sqrt{\pi}\left(\frac{\tau_{r}(\kappa)}{q_{r}(\kappa)}\right)^{1 / 4} \\
& \times\left[\left(\frac{1}{6} \frac{\frac{\partial}{\partial r} \Psi_{r}(\kappa)}{\Psi_{r}(\kappa)}-\frac{1}{4} \frac{\frac{\partial}{\partial r} q_{r}(\kappa)}{q_{r}(\kappa)}\right) \operatorname{Ai}\left(-\tau_{r}(\kappa)\right)-\frac{2}{3} \frac{\frac{\partial}{\partial r} \Psi_{r}(\kappa)}{\Psi_{r}(\kappa)} \tau_{r}(\kappa) \mathrm{Ai}^{\prime}\left(-\left(\tau_{r}(\kappa)\right)\right] \mathbb{1}_{\mathscr{K}},\right.
\end{aligned}
$$

and

$$
\begin{aligned}
\mathbb{B}(r, \kappa)=\frac{1}{1+\mathrm{i}}\left(-\frac{\frac{\partial}{\partial r} q_{r}(\kappa)}{4 q_{r}(\kappa)}\right. & \left.-\mathrm{i} \omega \sqrt{q_{r}(\kappa)}\right)\left(q_{r}(\kappa)\right)^{-1 / 4} e^{-\mathrm{i} \omega \psi_{r}(\kappa)} \mathbb{1}_{\mathscr{K}^{C}}+\sqrt{\pi}\left(\frac{\tau_{r}(\kappa)}{q_{r}(\kappa)}\right)^{1 / 4} \\
\times & {\left[\left(\frac{1}{6} \frac{\frac{\partial}{\partial r} \Psi_{r}(\kappa)}{\Psi_{r}(\kappa)}-\frac{1}{4} \frac{\frac{\partial}{\partial r} q_{r}(\kappa)}{q_{r}(\kappa)}\right) \operatorname{Bi}\left(-\tau_{r}(\kappa)\right)-\frac{2}{3} \frac{\frac{\partial}{\partial r} \Psi_{r}(\kappa)}{\Psi_{r}(\kappa)} \tau_{r}(\kappa) \operatorname{Bi}^{\prime}\left(-\left(\tau_{r}(\kappa)\right)\right] \mathbb{1}_{\mathscr{K}} .\right.}
\end{aligned}
$$

The region $\mathscr{K}$ is the region of $\kappa$ space where we have single zero of $q_{n}(r, \kappa)$ near the duct, and is defined by $\mathscr{K}=s^{+}(\mathscr{R}) \cup s^{-}(\mathscr{R})$ where

$$
s^{ \pm}(r)=\frac{U_{x}(r)\left(1-\frac{\eta U_{\theta}(r)}{r}\right) \pm c_{0}(r) \sqrt{\left(1-\frac{\eta U_{\theta}(r)}{r}\right)^{2}+\frac{\eta^{2}}{r^{2}}\left[U_{x}^{2}(r)-c_{0}^{2}(r)\right]}}{U_{x}^{2}(r)-c_{0}^{2}(r)} .
$$

Some examples of the region $\mathscr{K}$ are given in Mathews and Peake [26].

When $q_{n}(r, \kappa)$ has more than one zero we have to solve numerically for $\mathscr{A}(r, \kappa), \mathscr{B}(r, \kappa), \mathbb{A}(r, \kappa)$ and $\mathbb{B}(r, \kappa)$, with the method described in Mathews and Peake [26.

\section{Appendix B. Comparison of source terms in acoustic analogy}

In this section we show that

$$
\mathbb{S}_{1}^{M}=\mathbb{S}_{1}^{P P}+\mathbb{S}_{F W H, 1}^{P P} \text { and } \mathbb{S}_{2}^{M}=\mathbb{S}_{2}^{P P}+\mathbb{S}_{F W H, 2}^{P P},
$$

where the source terms with a ${ }^{P P}$ superscript are defined in Posson and Peake [32, Equation 3.9,3.10]. The source terms in this paper are given by

$$
\mathbb{S}_{1}^{M}=\frac{\overline{D_{0}}}{D t}\left(\mathcal{S}_{r}\right)+\frac{U_{\theta}^{2}}{r}\left(\mathcal{S}_{\text {mass }}-\frac{\mathcal{S}_{e}}{c_{0}^{2}}\right)+\frac{2 U_{\theta}}{r} \mathcal{S}_{\theta}+\frac{\overline{D_{0}}}{D t}\left(\mathcal{D}_{r}\right)+\frac{U_{\theta}^{2}}{r}\left(\mathcal{D}_{\text {mass }}-\frac{\mathcal{D}_{e}}{c_{0}^{2}}\right)+\frac{2 U_{\theta}}{r} \mathcal{D}_{\theta},
$$


and

$$
\mathbb{S}_{2}^{M}=\frac{\overline{D_{0}}}{D t}\left(\frac{\mathcal{S}_{e}}{c_{0}^{2}}\right)-\frac{\bar{\partial}}{\partial x}\left(\mathcal{S}_{x}\right)-\frac{1}{r} \frac{\bar{\partial}}{\partial \theta} \mathcal{S}_{\theta}+\frac{\overline{D_{0}}}{\overline{D t}}\left(\frac{\mathcal{D}_{e}}{c_{0}^{2}}\right)-\frac{\bar{\partial}}{\partial x}\left(\mathcal{D}_{x}\right)-\frac{1}{r} \frac{\bar{\partial}}{\partial \theta} \mathcal{D}_{\theta},
$$

while the source terms from Posson and Peake are given by

$$
\mathbb{S}_{1}^{P P}+\mathbb{S}_{F W H, 1}^{P P}=\frac{\overline{D_{0}}}{D t}\left(\mathcal{S}_{r}^{P P}\right)+\frac{2 U_{\theta}}{r} \mathcal{S}_{\theta}^{P P}+\frac{\overline{D_{0}}}{D t}\left(\mathcal{D}_{r}^{P P}\right)+\frac{2 U_{\theta}}{r} \mathcal{D}_{\theta}^{P P}
$$

and

$$
\mathbb{S}_{2}^{P P}+\mathbb{S}_{F W H, 2}^{P P}=\frac{\overline{D_{0}}}{D t}\left(\mathcal{S}_{\rho}^{P P}\right)-\frac{\bar{\partial}}{\partial x}\left(\mathcal{S}_{x}^{P P}\right)-\frac{1}{r} \frac{\bar{\partial}}{\partial \theta} \mathcal{S}_{\theta}^{P P}+\frac{\overline{D_{0}}}{D t}\left(\mathcal{D}_{\rho}^{P P}\right)-\frac{\bar{\partial}}{\partial x}\left(\mathcal{D}_{x}^{P P}\right)-\frac{1}{r} \frac{\bar{\partial}}{\partial \theta} \mathcal{D}_{\theta}^{P P} .
$$

We have the relations Eq. (27) to Eq. (31),

$$
\mathcal{S}_{\rho}^{P P}=\mathcal{S}_{\text {mass }}+\frac{\overline{D_{0}} \widetilde{Z_{p}}}{D t}, \quad \mathcal{S}^{P \boldsymbol{P}}=\mathcal{S}-\frac{U_{\theta}^{2}}{r_{0}} \widetilde{Z_{p}} \boldsymbol{e}_{\boldsymbol{r}}
$$

and

$$
\mathcal{D}_{\rho}^{P P}=\mathcal{D}_{\text {mass }}, \quad \mathcal{D}^{P P}=\mathcal{D},
$$

where $Z_{\mathrm{NI}}=p / c_{0}^{2}-\rho$ is the non-isentropic factor defined in Posson and Peake 32] (which is zero to leading order). Using the mass and energy equation, we can calculate the material derivative of $Z_{\mathrm{NI}}$ as

$$
\frac{D_{0} Z_{\mathrm{NI}}}{D t}=\boldsymbol{\nabla} \cdot(\rho \boldsymbol{u})-\frac{1}{c_{0}^{2}}(\boldsymbol{u} \cdot \nabla p+\gamma p(\boldsymbol{\nabla} \cdot \boldsymbol{u}))
$$

Converting this equation into an equation for generalised functions gives

$$
\frac{\overline{D_{0}} \widetilde{Z_{\mathrm{NI}}}}{D t}-Z_{\mathrm{NI}}\left(\boldsymbol{u}_{\mathbf{0}}-\boldsymbol{v}^{\mathcal{S}}\right) \cdot \boldsymbol{n} \delta\left(f_{\mathcal{S}}\right)=\bar{\nabla} \cdot(\widetilde{\rho} \widetilde{\boldsymbol{u}})-\frac{1}{c_{0}^{2}}(\widetilde{\boldsymbol{u}} \cdot \bar{\nabla} \widetilde{p}-\gamma \tilde{p} \bar{\nabla} \cdot \widetilde{\boldsymbol{u}})+Z_{\mathrm{NI}} \boldsymbol{u} \cdot \boldsymbol{n} \delta\left(f_{\mathcal{S}}\right)+\frac{\gamma p}{c_{0}^{2}} \boldsymbol{u} \cdot \boldsymbol{n} \delta\left(f_{\mathcal{S}}\right)
$$

First source term We now calculate that

$$
\begin{aligned}
\overline{\frac{D_{0}}{D t}}\left(\mathcal{S}_{r}\right)+\frac{U_{\theta}^{2}}{r} & \left(\mathcal{S}_{\text {mass }}-\frac{\mathcal{S}_{e}}{c_{0}^{2}}\right)+\frac{U_{\theta}^{2}}{r}\left(\mathcal{D}_{\text {mass }}-\frac{\mathcal{D}_{e}}{c_{0}^{2}}\right) \\
& =\frac{\overline{D_{0}}}{D t}\left(\mathcal{S}_{r}\right)-\frac{U_{\theta}^{2}}{r} \frac{\overline{D_{0}} \widetilde{Z_{\mathrm{NI}}}}{D t}+\frac{U_{\theta}^{2}}{r}\left[Z_{\mathrm{NI}}\left(\underline{\boldsymbol{u}}-\boldsymbol{v}^{\mathcal{S}}\right) \cdot \boldsymbol{n}+\frac{\gamma p}{c_{0}^{2}} \boldsymbol{u} \cdot \boldsymbol{n}\right] \delta\left(f_{\mathcal{S}}\right) \\
& +\frac{U_{\theta}^{2}}{r}\left[\left(\rho\left(\underline{\boldsymbol{u}}-\boldsymbol{v}^{\mathcal{S}}\right) \cdot \boldsymbol{n}+\rho_{0}(\boldsymbol{u} \cdot \boldsymbol{n})\right)-\frac{1}{c_{0}^{2}}\left(p\left(\underline{\boldsymbol{u}}-\boldsymbol{v}^{\mathcal{S}}\right) \cdot \boldsymbol{n}+\gamma p_{0}(\boldsymbol{u} \cdot \boldsymbol{n})+\gamma p(\boldsymbol{u} \cdot \boldsymbol{n})\right)\right] \delta\left(f_{\mathcal{S}}\right) \\
& =\frac{\overline{D_{0}}}{D t}\left(\mathcal{S}_{r}\right)-\frac{U_{\theta}^{2}}{r} \frac{\overline{D_{0}}}{D Z_{\mathrm{NI}}}=\frac{\overline{D_{0}}}{D t}\left(\mathcal{S}_{r}^{P P}\right) .
\end{aligned}
$$

Hence, we have $\mathbb{S}_{1}^{M}=\mathbb{S}_{1}^{P P}+\mathbb{S}_{F W H, 1}^{P P}$.

Second source term Finally, we calculate that

$$
\begin{aligned}
\frac{\overline{D_{0}}}{D t}\left(\frac{\mathcal{S}_{e}}{c_{0}^{2}}\right)+\frac{\overline{D_{0}}}{D t}\left(\frac{\mathcal{D}_{e}}{c_{0}^{2}}\right) & =\frac{\overline{D_{0}}}{D t}\left(-\bar{\nabla} \cdot(\widetilde{\rho} \widetilde{\boldsymbol{u}})+\frac{\overline{D_{0}} \widetilde{Z_{\mathrm{NI}}}}{D t}-\left[Z_{\mathrm{NI}}\left(\underline{\boldsymbol{u}}+\boldsymbol{v}^{\mathcal{S}}\right) \cdot \boldsymbol{n}+\frac{\gamma p}{c_{0}^{2}}(\boldsymbol{u} \cdot \boldsymbol{n})\right] \delta\left(f_{\mathcal{S}}\right)\right) \\
& +\frac{\overline{D_{0}}}{\overline{D t}}\left(\left[\frac{p}{c_{0}^{2}}\left(\underline{\boldsymbol{u}}-\boldsymbol{v}^{\mathcal{S}}\right) \cdot \boldsymbol{n}+\frac{\gamma p_{0}}{c_{0}^{2}} \boldsymbol{u} \cdot \boldsymbol{n}+\frac{\gamma p}{c_{0}^{2}} \boldsymbol{u} \cdot \boldsymbol{n}\right] \delta\left(f_{\mathcal{S}}\right)\right) \\
& =\frac{\overline{D_{0}}}{D t}\left(-\bar{\nabla} \cdot(\widetilde{\rho} \widetilde{\boldsymbol{u}})+\left[\rho\left(\underline{\boldsymbol{u}}-\boldsymbol{v}^{\mathcal{S}}\right) \cdot \boldsymbol{n}+\rho_{0} \boldsymbol{u} \cdot \boldsymbol{n}\right] \delta\left(f_{\mathcal{S}}\right)\right)=\frac{\overline{D_{0}}}{D t}\left(\mathcal{S}_{\rho}^{P P}\right)+\frac{\overline{D_{0}}}{D t}\left(\mathcal{D}_{\rho}^{P P}\right) .
\end{aligned}
$$

Hence, we have $\mathbb{S}_{2}^{M}=\mathbb{S}_{2}^{P P}+\mathbb{S}_{F W H, 2}^{P P}$ and the source terms from the analogy in this paper and the analogy in Posson and Peake [32] are equal for a homentropic flow. 


\section{References}

[1] C. M. Bender and S. A. Orszag. Advanced mathematical methods for scientists and engineers I: Asymptotic methods and perturbation theory. Springer, 1978.

[2] W. E. Boyce, R. C. DiPrima, and C. W. Haines. Elementary differential equations and boundary value problems. Wiley New York, 1992.

[3] E. J. Brambley and N. Peake. Classification of aeroacoustically relevant surface modes in cylindrical lined ducts. Wave Motion, 43(4):301-310, 2006.

[4] J. Chen and P. Joseph. Measurement techniques for mode detection in aeroengine inter-stage sections. In 22nd AIAA/CEAS Aeroacoustics Conference, Lyon, 2016.

[5] A. J. Cooper. Effect of mean entropy on unsteady disturbance propagation in a slowly varying duct with mean swirling flow. Journal of Sound and Vibration, 291(3):779-801, 2006.

[6] A. J. Cooper and N. Peake. Upstream-radiated rotor-stator interaction noise in mean swirling flow. Journal of Fluid Mechanics, 523:219-250, 2005.

[7] D. G. Crighton, A. P. Dowling, J. E. Ffowcs Williams, M. Heckl, and F. G. Leppington. Modern methods in analytical acoustics lecture notes. Springer-Verlag, 1992.

[8] N. Curle. The influence of solid boundaries upon aerodynamic sound. In Proceedings of the Royal Society of London A: Mathematical, Physical and Engineering Sciences, volume 231, pages 505-514. The Royal Society, 1955.

[9] T. A. Driscoll, N. Hale, and L. N. Trefethen. Chebfun Guide. Pafnuty Publications, Oxford, 2014.

[10] W. Eversman and R. J. Beckemeyer. Transmission of sound in ducts with thin shear layersconvergence to the uniform flow case. The Journal of the Acoustical Society of America, 52(1B):216-220, 1972.

[11] F. Farassat. Introduction to generalized functions with applications in aerodynamics and aeroacoustics. NASA Technical Paper 3428, 1996.

[12] J. E. Ffowcs Williams and D. L. Hawkings. Sound generation by turbulence and surfaces in arbitrary motion. Philosophical Transactions of the Royal Society of London. Series A, Mathematical and Physical Sciences, 264(1151):321-342, 1969.

[13] M. E. Goldstein. An exact form of Lilley's equation with a velocity quadrupole/temperature dipole source term. Journal of Fluid Mechanics, 443:231-236, 2001.

[14] V. V. Golubev and H. M. Atassi. Acoustic-vorticity waves in swirling flows. Journal of Sound and Vibration, 209:203-222, 1998.

[15] G. Grubb. Distributions and operators. Springer, 2009.

[16] C. J. Heaton and N. Peake. Acoustic scattering in a duct with mean swirling flow. Journal of Fluid Mechanics, 540: 189-220, 2005.

[17] C. J. Heaton and N. Peake. Algebraic and exponential instability of inviscid swirling flow. Journal of Fluid Mechanics, 565:279-318, 2006

[18] P. Joseph, C. L. Morfey, and C. R. Lowis. Multi-mode sound transmission in ducts with flow. Journal of Sound and Vibration, 264(3):523-544, 2003.

[19] J. L. Kerrebrock. Small disturbances in turbomachine annuli with swirl. AIAA Journal, 15(6):794-803, 1977.

[20] D. P. Lalas. The "Richardson" criterion for compressible swirling flows. Journal of Fluid Mechanics, 69:65-72, 1975.

[21] M. J. Lighthill. On sound generated aerodynamically. I. General theory. In Proceedings of the Royal Society of London A: Mathematical, Physical and Engineering Sciences, volume 211, pages 564-587. The Royal Society, 1952.

[22] G. M. Lilley. On the noise from jets. AGARD Technical Report CP-131, pages 13.1-13.12, 1974.

[23] V. Masson, H. Posson, M. Sanjose, S. Moreau, and M. Roger. Fan-OGV interaction broadband noise prediction in a rigid annular duct with swirling and sheared mean flow. In 22nd AIAA/CEAS Aeroacoustics Conference, Lyon, 2016.

[24] V. Masson, J. R. Mathews, H. Posson, M. Sanjose, and S. Moreau. Liner behavior in an annular duct with swirling and sheared mean flow. In 23rd AIAA/CEAS Aeroacoustics Conference, Denver, 2017.

[25] J. R. Mathews. Mathematical modelling of noise generation in turbofan aeroengines using Green's functions. PhD thesis, University of Cambridge, 2016. URL http://dx.doi.org/10.17863/CAM.8962.

[26] J. R. Mathews and N. Peake. The acoustic Green's function for swirling flow in a lined duct. Journal of Sound and Vibration, 395:294-316, 2017.

[27] J. R. Mathews, N. Peake, and S. Bianchi. Asymptotic and numerical Green's function in a lined duct with realistic shear and swirl. In 22nd AIAA/CEAS Aeroacoustics Conference, Lyon, 2016.

[28] C. L. Morfey and M. C. M. Wright. Extensions of Lighthill's acoustic analogy with application to computational aeroacoustics. In Proceedings of the Royal Society of London A: Mathematical, Physical and Engineering Sciences, volume 463, pages 2101-2127. The Royal Society, 2007.

[29] M. K. Myers. On the acoustic boundary condition in the presence of flow. Journal of Sound and Vibration, 71(3):429-434, 1980.

[30] R. Nijboer. Eigenvalues and eigenfunctions of ducted swirling flows. In 7th AIAA/CEAS Aeroacoustics Conference, Maastricht, 2001.

[31] H. Posson and N. Peake. Swirling mean flow effect on fan-trailing edge broadband noise in a lined annular duct. In 19th AIAA/CEAS Aeroacoustics Conference, Berlin, 2013.

[32] H. Posson and N. Peake. The acoustic analogy in an annular duct with swirling mean flow. Journal of Fluid Mechanics, 726:439-475, 2013.

[33] D. C. Pridmore-Brown. Sound propagation in a fluid flowing through an attenuating duct. The Journal of the Acoustical Society of America, 30(7):670-670, 1958.

[34] S. W. Rienstra. A classification of duct modes based on surface waves. Wave Motion, 37(2):119-135, 2003. 
[35] P. Sijtsma. Using phased array beamforming to locate broadband noise sources inside a turbofan engine. In AARC Engine Noise Phased Array Workshop, Cambridge (MA), 2006.

[36] P. Sijtsma. Green's functions for in-duct beamforming applications. In 18th AIAA/CEAS Aeroacoustics Conference, Colorado Springs, volume 2248, 2012.

[37] C. K. W. Tam and L. Auriault. The wave modes in ducted swirling flows. Journal of Fluid Mechanics, 371:1-20, 1998.

[38] G. G. Vilenski and S. W. Rienstra. On hydrodynamic and acoustic modes in a ducted shear flow with wall lining. Journal of Fluid Mechanics, 583:45-70, 2007.

[39] G. G Vilenski and S. W. Rienstra. Numerical study of acoustic modes in ducted shear flow. Journal of Sound and Vibration, 307(3):610-626, 2007. 